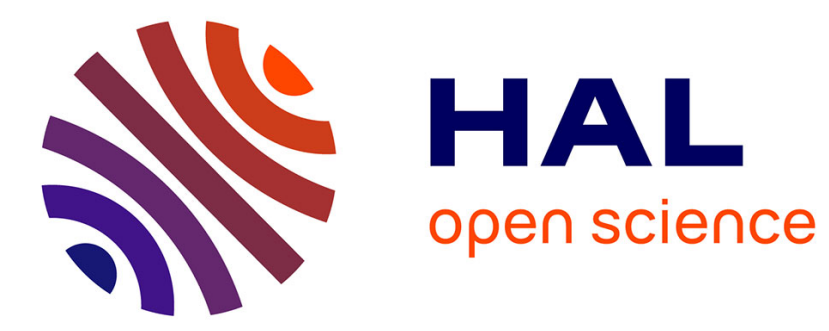

\title{
Some new results on generalized additive games
}

Stefano Moretti, Henk Norde

\section{- To cite this version:}

Stefano Moretti, Henk Norde. Some new results on generalized additive games. International Journal of Game Theory, Springer Verlag, 2021, 10.1007/s00182-021-00786-w . hal-03388816

\section{HAL Id: hal-03388816 \\ https://hal.archives-ouvertes.fr/hal-03388816}

Submitted on 20 Oct 2021

HAL is a multi-disciplinary open access archive for the deposit and dissemination of scientific research documents, whether they are published or not. The documents may come from teaching and research institutions in France or abroad, or from public or private research centers.
L'archive ouverte pluridisciplinaire HAL, est destinée au dépôt et à la diffusion de documents scientifiques de niveau recherche, publiés ou non, émanant des établissements d'enseignement et de recherche français ou étrangers, des laboratoires publics ou privés. 


\title{
Some new results on generalized additive games
}

\author{
Stefano Moretti ${ }^{1}$ and Henk Norde ${ }^{2}$
}

\begin{abstract}
A Generalized Additive Game (GAG) [9] is a Transferable Utility (TU) game $(N, v)$, where each player in $N$ is provided with an individual value, and the worth $v(S)$ of a coalition $S \subseteq N$ is obtained as the sum of the individual values of players in another subset $\mathcal{M}(S) \subseteq N$. Based on conditions on the map $\mathcal{M}$ (which associates to each coalition $S$ a set of beneficial players $\mathcal{M}(S)$ not necessarily included in $S$ ), in this paper we characterize classes of GAGs that satisfy properties like monotonicity, superadditivity, (total) balancedness, PMAS-admissibility and supermodularity, for all nonnegative vectors of individual values. We also illustrate the application of such conditions on $\mathcal{M}$ over particular GAGs studied in the literature (e.g., glove games [24], generalized airport games [20], fixed tree games [4], link-connection games [19, 16], simple minimum cost spanning tree games [21, 27] and graph coloring games $[10,11])$.
\end{abstract}

Keywords: TU-games, monotonicity, balancedness, population monotonic allocation scheme (PMAS), supermodularity, operations research games.

\section{Introduction}

Generalized additive games (GAGs) are Transferable Utility (TU) games, where the worth of any coalition of players can be computed as a sum of individual contributions. As shown in [9], many TU games from the literature can be represented as GAGs, like, for instance, airport games [14, 15], connectivity games [2, 13], argumentation games [5], centrality games [26, 1], peer games [7], games on mountain situations [18], etc.

A basic ingredient for GAGs is the so called coalitional map [9], that specifies the set of friends (or contributors) of a coalition $S \subseteq N$, where $N=\{1, \ldots, n\}$ is a finite set of players. Given a coalitional map and a vector of $n$ nonnegative real numbers representing the individual contribution of players in $N$, the corresponding GAG assigns to every coalition $S \subseteq N$ the sum of the contributions over the set of friends of $S$. In this paper we study the effect of the combination of four properties for coalitional maps on the corresponding GAGs engendered by any vector of nonnegative individual contributions. To be more specific, the first property we introduce is a monotonicity condition: a coalitional map is called monotonic if the friends of any coalition $S \subseteq N$ are also friends of any coalition $T$ containing $S$. Differently, a coalitional map is called proper if any two disjoint coalitions $S, T \subseteq N, S \cap T=\emptyset$, have no friends in common. The third property deals with the veto-behavior of players. More precisely, a coalitional map is called veto-rich if for any player $i \in N$, either $i$ is not a friend of any coalition, or $i$ is a friend of the grand coalition $N$ and, at the same time, the intersection of coalitions having $i$ as a friend is non-empty. Finally, a coalitional map is supermodular if the intersection of the set of friends of two coalitons $S, T \subseteq N$ coincides with the set of friends of their intersection. We first prove that these properties for coalitional maps characterize some interesting classes of GAGs as follows: 1) monotonicity of a coalitional map is equivalent to monotonicity of the corresponding GAGs; 2) monotonicity and properness of a coalitional map is equivalent to superadditivity of the corresponding GAGs; 3) veto-richness of a coalitional map is equivalent to balancedness of the corresponding GAGs; 4) monotonicity and veto-richness of a coalitional map is equivalent to both total balancedness and admissibility of a population monotonic allocation scheme (PMAS) [25] of the corresponding GAGs; 5) supermodularity of a coalitional map is equivalent to supermodularity or convexity of the corresponding GAGs. Then, we use these characterizations to analyze several classes of TU games from the literature, with a particular focus on Operations Research (OR) games [6]. In particular, we consider (weighted) glove games [24, 28], (generalized) airport games [20], fixed tree games [4], link-connection games $[19,16]$, simple minimum cost spanning tree games (MCST) [21, 27] and (weighted) coloring games $[10,11]$.

A main advantage of representing and studying a TU game as a GAG lies in the combinatorial nature of the four properties proposed for coalitional maps. In all classes of TU games from the literature considered in this paper, it is straightforward to verify whether the properties of monotonicity, properness, veto-richness and supermodularity

\footnotetext{
${ }^{1}$ Université Paris-Dauphine, PSL Research University, CNRS, UMR [7243], LAMSADE, 75016 Paris, France. Email: stefano.moretti@dauphine.fr

${ }^{2}$ CentER and Department of Econometrics and Operations Research, Tilburg University, P.O. Box 90153,5000 LE Tilburg, The Netherlands, Email: h.norde@tilburguniversity.edu
} 
hold for the corresponding coalitional maps. So, the method of proof that we propose in this paper is much less time-consuming than the techniques adopted in the related literature. Moreover, some nice properties of certain well-known TU games, can be easily generalized over larger domains, or they can be used to investigate in more details some particular sub-classes of games. As a simple example, consider the case of airport games [14, 15], and the corresponding cost saving game, where the costs of the airport's runway is supposed to increase with the length of the landing strip, i.e. the cost vector $w$ belongs to the convex cone $K_{1} \subset \mathbb{R}_{+}^{N}$ defined by $K_{1}=\left\{w \in \mathbb{R}_{+}^{N}: w_{1} \leq\right.$ $\left.w_{2} \leq \cdots \leq w_{n}\right\}$. Looking at the corresponding cost saving game, and using our characterizations, the properties of monotonicity, superadditivity, balancedness and PMAS-admissibility can be extended to airport games associated to any cost vector in $\mathbb{R}_{+}^{N}$, and in particular to the larger cone $K_{2}=\left\{w \in \mathbb{R}_{+}^{N}: w_{i} \leq w_{n}\right.$ for every $\left.i \in\{1, \ldots, n-1\}\right\}$, whose family of corresponding GAGs coincides with the class of generalized airport games introduced in [20].

We also stress the fact that all of our results hold for profit games, i.e. for GAGs whose individual contributions can be interpreted as revenues or profits. We adopted this convention for consistency with the interpretation of a coalitional map as a specification of friends of coalitions, and for coherence with the more popular interpretation of TU games as revenue-sharing situations, as presented in the original paper [9]. On the other hand, the majority of games we consider in this paper deal with cost sharing situations. Following a standard approach, we consider the corresponding cost saving game, that is a TU game where the worth of each coalition $S$ represents the amount that coalition $S$ saves by cooperation, and is computed as the difference between the sum of the costs of singleton coalitions formed by the members of $S$ minus the total cost of coalition $S$. In alternative, another standard way to transform a cost game into a profit one, is by means of the corresponding dual game, where a coalition $S$ gets the rest of the cost of the grand coalition $N$ after the complement of coalition $S$ pays its entire cost in the original game. So, the dual game of a cost game can be interpreted as the opportunity for players in $S$ to fully profit of the contribution of players outside $S$.

The road-map of the paper is as follows. We start with some preliminary notions and notations on game theory and graph theory in Section 2. In Section 3 we introduce some properties for coalitional maps and we illustrate some characterizations of GAGs using (combinations of) these properties. Then, in Section 4, we analyze several classes of TU games from the literature that can be represented as GAGs and can be studied in view of the results presented in Section 3. Specifically, we consider weighted glove games in Section 4.1, generalized airport games in Section 4.2, fixed tree games in Section 4.3, link connection games in Section 4.4, simple MCST games in Section 4.5 and (weighted) coloring games in Section 4.6. Section 5 concludes.

\section{Preliminaries and notations}

\subsection{Game theory}

A Transferable Utility (TU) game (or, simply, a game) is a pair $(N, v)$, where $N=\{1, \ldots, n\}$ denotes the set of players, and $v: 2^{N} \rightarrow \mathbb{R}$ is the characteristic function that maps each element of $2^{N}$ (the set of all subsets of $N$ ) to a real number (by convention, $v(\emptyset)=0$ ). For each $S \in 2^{N}, v(S)$ represents the worth or profit of coalition $S$. In the following, we often identify a game $(N, v)$ with its characteristic function $v$ (if the set $N$ is fixed). Some basic properties for TU games are introduced in the next definition.

Definition 2.1 A game $(N, v)$ is called:

1) monotonic if $v(S) \leq v(T)$ for all $S, T \in 2^{N}$ such that $S \subseteq T$;

2) superadditive if $v(S \cup T) \geq v(S)+v(T)$ for all $S, T \in 2^{N}$ such that $S \cap T=\emptyset$;

3) supermodular or convex if $v(S \cup T)+v(S \cap T) \geq v(S)+v(T)$ for all $S, T \in 2^{N}$ or, equivalently, $v(S \cup\{i\})-v(S) \leq$ $v(T \cup\{i\})-v(T)$ for all $i \in N$ and all $S \subseteq T \subseteq N \backslash\{i\}$.

A game $(N, v)$ such that $v(S) \in\{0,1\}$ (i.e., the worth of every coalition is either 0 or 1 ) for each $S \in 2^{N}$ and $v(N)=1$ is said to be a simple game. A player $i \in N$ in a simple game $(N, v)$ is called a veto player if any $S \in 2^{N}$ with $v(S)=1$ satisfies $i \in S$. 
Given a game $(N, v)$, an allocation is a vector $x \in \mathbb{R}^{N}$, and an allocation $x \in \mathbb{R}^{N}$ is in the core $C(v)$ of game $v$ if it is efficient (i.e., $\sum_{i \in N} x_{i}=v(N)$ ) and stable (i.e., $\sum_{i \in S} x_{i} \geq v(S)$ for all non-empty coalitions $S \in 2^{N}$ ). So,

$$
C(v)=\left\{x \in \mathbb{R}^{N}: \sum_{i \in N} x_{i}=v(N), \sum_{i \in S} x_{i} \geq v(S), \text { for all } S \in 2^{N}, S \neq \emptyset\right\} .
$$

A game $v$ such that $C(v) \neq \emptyset$ is called balanced. Given a game $(N, v)$ and a coalition $S \in 2^{N}$, we denote by $\left(S, v_{\mid S}\right)$ the subgame of $v$ restricted to coalition $S$ such that $v_{\mid S}(R)=v(R)$ for each $R \subseteq S$. A game $v$ such that $C\left(v_{\mid S}\right) \neq \emptyset$ for all $S \in 2^{N}$ is called totally balanced.

A population monotonic allocation scheme or PMAS [25] of the game $(N, v)$ is a scheme $\left\{x_{S, i}\right\}_{S \in 2^{N} \backslash\{\emptyset\}, i \in S}$ with the properties:

i) $\sum_{i \in S} x_{S, i}=v(S)$ for all $S \in 2^{N} \backslash\{\emptyset\}$ (efficiency);

ii) $x_{S, i} \leq x_{T, i}$ for all $S, T \in 2^{N} \backslash\{\emptyset\}$ and $i \in N$ with $i \in S \subset T$ (monotonicity).

A PMAS provides an allocation vector for every coalition in a monotonic way, i.e. the value allocated to some player increases if the coalition to which he belongs becomes larger. If a game $v$ has a PMAS, then it is said to be PMAS-admissible. It is easy to check that a PMAS $\left\{x_{S, i}\right\}_{S \in 2^{N} \backslash\{\emptyset\}, i \in S}$ provides a core element for the game and all its subgames, i.e. the allocation $\left(x_{S, i}\right)_{i \in S} \in C\left(v_{\mid S}\right)$ for all $S \in 2^{N}, S \neq \emptyset$. Therefore, a game admitting a PMAS is also totally balanced.

\subsection{Generalized Additive Games (GAGs)}

We now introduce some basic definitions on GAGs from [9]. A coalitional map is a map $\mathcal{M}: 2^{N} \rightarrow 2^{N}$ such that $\mathcal{M}(\emptyset)=\emptyset$. The elements of $\mathcal{M}(S)$, for each $S \in 2^{N}$, are called friends or contributors of $S$ via $\mathcal{M}$.

Definition 2.2 $A$ generalized additive situation (GAS)[9] is a triple $(N, \mathcal{M}, w)$, where $N$ is a finite set of players, $\mathcal{M}$ is a coalitional map and $w \in \mathbb{R}_{+}^{N}$ is a vector of nonnegative real numbers $w_{i} \geq 0$, for any $i \in N$. The corresponding generalized additive game $(\mathrm{GAG})\left(N, v_{\mathcal{M}, w}\right)$ is defined by

$$
v_{\mathcal{M}, w}(S)=\sum_{i \in \mathcal{M}(S)} w_{i}
$$

for every $S \in 2^{N}$.

So, in order to compute the worth of a coalition $S \in 2^{N}$ in a $\operatorname{GAG}\left(N, v_{\mathcal{M}, w}\right)$, we first select the friends of $S$, i.e. the players in $\mathcal{M}(S)$, and then we add their individual values according to $w$. Given a coalitional map $\mathcal{M}$ on $2^{N}$, the class of all GAGs $\left(N, v_{\mathcal{M}, w}\right)$, for any $w \in \mathbb{R}_{+}^{N}$, is denoted by $\mathcal{G}^{\mathcal{M}}$.

It is straightforward to see that a $\operatorname{GAG}\left(N, v_{\mathcal{M}, w}\right)$ is a nonnegative combination of simple games $\left(N, v_{\mathcal{M}, i}\right)$,

$$
v_{\mathcal{M}, w}=\sum_{i \in N} w_{i} v_{\mathcal{M}, i}
$$

where for every $i \in N$ the simple game $\left(N, v_{\mathcal{M}, i}\right)$ is defined by

$$
v_{\mathcal{M}, i}(S)= \begin{cases}1 & \text { if } i \in \mathcal{M}(S) \\ 0 & \text { if } i \notin \mathcal{M}(S)\end{cases}
$$

for every $S \in 2^{N}$. So the winning coalitions in $\left(N, v_{\mathcal{M}, i}\right)$ are precisely those coalitions that select player $i$. Note that $\left(N, v_{\mathcal{M}, i}\right)$ can also be regarded as the GAG corresponding to coalitional map $\mathcal{M}$ and unit weight vector $e^{i} \in\{0,1\}^{N}$ $\left(e_{i}^{i}=1\right.$ and $e_{j}^{i}=0$ if $\left.j \neq i\right)$. 
For each $S \in 2^{N}$, we denote by $\left(S,\left(v_{\mathcal{M}, w}\right)_{\mid S}\right)$ and $\left(S,\left(v_{\mathcal{M}, i}\right)_{\mid S}\right)$ the restrictions to $S$ of games $\left(N, v_{\mathcal{M}, w}\right)$ and $\left(N, v_{\mathcal{M}, i}\right)$, respectively, i.e., $\left(v_{\mathcal{M}, w}\right)_{\mid S}(R)=v_{\mathcal{M}, w}(R)$ and $\left(v_{\mathcal{M}, i}\right)_{\mid S}(R)=v_{\mathcal{M}, i}(R)$ for each $R \subseteq S$. It is straightforward to see that the subgame $\left(S,\left(v_{\mathcal{M}, w}\right)_{\mid S}\right)$ is a nonnegative combination of simple subgames $\left(S,\left(v_{\mathcal{M}, i}\right)_{\mid S}\right), i \in N$, precisely,

$$
\left(v_{\mathcal{M}, w}\right)_{\mid S}=\sum_{i \in N} w_{i}\left(v_{\mathcal{M}, i}\right)_{\mid S}
$$

for every $S \in 2^{N}$.

\subsection{Graph theory}

An (undirected) graph is a pair $\Gamma=(V, E)$, where $V$ is a set of vertices or nodes, and $E$ is a set of edges $e$ of the form $\{i, j\}$ with $i, j \in V, i \neq j$. The complete graph on a set $V$ of vertices is the graph $\left(V, E_{V}\right)$, where $E_{V}=\{\{i, j\} \mid i, j \in V$ and $i \neq j\}$. A path between two nodes $i, j \in V$ in a graph $\Gamma$ is a sequence of nodes $\left(i_{0}, i_{1}, \ldots\right.$, $\left.i_{k}\right)$, where $i=i_{0}$ and $j=i_{k}, k \geq 1$, such that $\left\{i_{s}, i_{s+1}\right\} \in E$ for each $s \in\{0, \ldots, k-1\}$ and such that all these edges are distinct. A cycle in $(V, E)$ is a path from $i$ to $i$ for some $i \in V$. A path $\left(i_{0}, i_{1}, \ldots, i_{k}\right)$ is without cycles if there do not exist $a, b \in\{0,1, \ldots, k\}, a \neq b$, such that $i_{a}=i_{b}$. Two nodes $i, j \in V$ are said to be connected in $\Gamma$ if $i=j$ or there exists a path between $i$ and $j$ in $\Gamma$. A graph $\Gamma$ is connected if for each $i, j \in V$ with $i \neq j$ there exists a path between $i$ and $j$ in $(V, E)$. A connected component of $\Gamma$ is a maximal subset of $V$ with the property that any two nodes in this subset are connected. A graph where all paths are without cycles is called a forest; a forest that is also connected is called a tree; a tree formed by a path containing all nodes is called a linear graph.

Given an undirected graph $\Gamma=(V, E)$, the subgraph $\Gamma_{\mid S}=\left(S, E_{S}\right)$ induced by $S \in 2^{V}$ is a graph with set $S$ as set of vertices and where $E_{S}=\{\{i, j\} \in E: i, j \in S\}$. A clique in $\Gamma$ is a subset $S \in 2^{V}$ such that $\Gamma_{\mid S}$ is complete.

\section{Some characterizations}

We start with the definition of some properties for coalitional maps.

Definition 3.1 A coalitional map $\mathcal{M}: 2^{N} \rightarrow 2^{N}$ is called:

1) monotonic if $\mathcal{M}(S) \subseteq \mathcal{M}(T)$ for every $S, T \in 2^{N}$ with $S \subseteq T$;

2) proper if $\mathcal{M}(S) \cap \mathcal{M}(T)=\emptyset$ for every $S, T \in 2^{N}$ with $S \cap T=\emptyset$;

3) veto-rich if for every $i \in N$ we either have $i \notin \mathcal{M}(S)$ for every $S \in 2^{N}$ or $i \in \mathcal{M}(N)$ and $\{S: i \in \mathcal{M}(S)\} \neq \emptyset$;

4) supermodular if $\mathcal{M}(S) \cap \mathcal{M}(T)=\mathcal{M}(S \cap T)$ for every $S, T \in 2^{N}$.

Properties in Definition 3.1 are naturally recast in terms of mechanisms aimed to select friends or contributors of coalitions. A coalitional map is called monotonic if it selects friends of coalitions in a monotonic way, i.e. each friend of a coalition is also a friend of any superset of that coalition. A coalitional map is called proper if two disjoint coalitions do not have a friend in common. A coalitional map is called veto-rich if it partitions the elements of $N$ in two particular subsets: the first subset includes those players that are never selected as friends of any coalition, whereas each player of the second subset is a friend of the grand coalition and, in addition, all coalitions with this player as a friend have a non-empty intersection. Finally, a coalitional map is supermodular, if the set of friends in common between any two coalitions coincides with the set of friends selected for their intersection.

Remark 3.2 Note that in [9], a map $\mathcal{M}$ is said to be proper if $\mathcal{M}(S) \subseteq S$ for all $S \in 2^{N}$. Clearly, the properness property introduced in [9] implies the properness property introduced in this section, but the implication in the other direction does not hold (for instance, take a coalitional map $\mathcal{M}$ with a nonempty image only for coalitions $\{1\}$ and $\{2\}$ and such that $\mathcal{M}(\{1\})=\{1,3\}$ and $\mathcal{M}(\{2\})=\{2,4\})$.

A natural question to ask is which properties for a coalitional map $\mathcal{M}$ induce nice game theoretical properties on the corresponding family of GAGs $\mathcal{G}^{\mathcal{M}}$. The following theorems provide necessary and sufficient conditions on 
a coalitional map $\mathcal{M}$ to generate corresponding GAGs $\left(N, v_{\mathcal{M}, w}\right)$ that are, respectively, monotonic, superadditive, (totally) balanced, PMAS-admissible and supermodular for every weight vector $w \in \mathbb{R}_{+}^{N}$.

The first theorem generalizes a result in [9], stating that a monotonic coalitional map induces a monotonic GAG (see Proposition 1 in [9]).

Theorem 3.3 $\mathcal{M}$ is monotonic if and only if $\left(N, v_{\mathcal{M}, w}\right)$ is monotonic for every $w \in \mathbb{R}_{+}^{N}$.

Proof " $\Rightarrow$ " Suppose $\mathcal{M}$ is monotonic. Let $w \in \mathbb{R}_{+}^{N}$ and $S, T \in 2^{N}$ with $S \subseteq T$. Then $\mathcal{M}(S) \subseteq \mathcal{M}(T)$, so $v_{\mathcal{M}, w}(S)=\sum_{i \in \mathcal{M}(S)} w_{i} \leq \sum_{i \in \mathcal{M}(T)} w_{i}=v_{\mathcal{M}, w}(T)$. So $v_{\mathcal{M}, w}$ is monotonic.

" $\Leftarrow$ " Suppose $\left(N, v_{\mathcal{M}, w}\right)$ is monotonic for every $w \in \mathbb{R}_{+}^{N}$. This implies, taking the unit weight vector $w=e^{i}$, that $\left(N, v_{\mathcal{M}, i}\right)$ is monotonic for every $i \in N$. Now let $S, T \in 2^{N}$ with $S \subseteq T$. For every $i \in \mathcal{M}(S)$ we have $1=v_{\mathcal{M}, i}(S) \leq v_{\mathcal{M}, i}(T)$ so $v_{\mathcal{M}, i}(T)=1$, and hence $i \in \mathcal{M}(T)$. So $\mathcal{M}(S) \subseteq \mathcal{M}(T)$. Therefore $\mathcal{M}$ is monotonic.

Also the following theorem generalizes a result in [9] (see Proposition 2 in [9]).

Theorem 3.4 $\mathcal{M}$ is monotonic and proper if and only if $\left(N, v_{\mathcal{M}, w}\right)$ is superadditive for every $w \in \mathbb{R}_{+}^{N}$.

Proof " $\Rightarrow$ " Suppose $\mathcal{M}$ is monotonic and proper. Let $w \in \mathbb{R}_{+}^{N}$ and $S, T \in 2^{N}$ with $S \cap T=\emptyset$. Due to monotonicity of $\mathcal{M}$ we have $\mathcal{M}(S) \subseteq \mathcal{M}(S \cup T)$ and $\mathcal{M}(T) \subseteq \mathcal{M}(S \cup T)$ so $\mathcal{M}(S) \cup \mathcal{M}(T) \subseteq \mathcal{M}(S \cup T)$. Due to properness of $\mathcal{M}$ we have $\mathcal{M}(S) \cap \mathcal{M}(T)=\emptyset$. Therefore $v_{\mathcal{M}, w}(S \cup \bar{T})=\sum_{i \in \mathcal{M}(S \cup T)} w_{i} \geq \sum_{i \in \mathcal{M}(S) \cup \mathcal{M}(T)} w_{i}=\sum_{i \in \mathcal{M}(S)} w_{i}+\sum_{i \in \mathcal{M}(T)} w_{i}=$ $v_{\mathcal{M}, w}(S)+v_{\mathcal{M}, w}(T)$. So $v_{\mathcal{M}, w}$ is superadditive.

" $\Leftarrow$ " Suppose $\left(N, v_{\mathcal{M}, w}\right)$ is superadditive for every $w \in \mathbb{R}_{+}^{N}$. Since GAGs are nonnegative it follows directly that $\left(N, v_{\mathcal{M}, w}\right)$ is monotonic for every $w \in \mathbb{R}_{+}^{N}$. From Theorem 3.3 it follows that $\mathcal{M}$ is monotonic. Again taking the unit weight vector $w=e^{i}$ we observe that in particular $\left(N, v_{\mathcal{M}, i}\right)$ is superadditive for every $i \in N$. Let $S, T \in 2^{N}$ with $S \cap T=\emptyset$. Suppose $\mathcal{M}(S) \cap \mathcal{M}(T) \neq \emptyset$ and let $i \in \mathcal{M}(S) \cap \mathcal{M}(T)$. Then $v_{\mathcal{M}, i}(S \cup T) \geq v_{\mathcal{M}, i}(S)+v_{\mathcal{M}, i}(T)=1+1=2$, contradicting the fact that $v_{\mathcal{M}, i}$ is a simple game. So $\mathcal{M}(S) \cap \mathcal{M}(T)=\emptyset$ and hence $\mathcal{M}$ is proper.

The next theorem deals with the notion of balancedness of TU games. Here, the veto-richness property for coalitional maps plays a central role.

Theorem 3.5 $\mathcal{M}$ is veto-rich if and only if $\left(N, v_{\mathcal{M}, w}\right)$ is balanced for every $w \in \mathbb{R}_{+}^{N}$.

Proof " $\Rightarrow$ " Suppose $\mathcal{M}$ is veto-rich. Let $i \in N$. If $i \notin \mathcal{M}(S)$ for every $S \in 2^{N}$, then $\left(N, v_{\mathcal{M}, i}\right)$ is the zero game and hence balanced. If $i \in \mathcal{M}(N)$ and $\cap\{S: i \in \mathcal{M}(S)\} \neq \emptyset$, then $v_{\mathcal{M}, i}(N)=1$, and there is a $j \in \cap\{S: i \in$ $\mathcal{M}(S)\}=\cap\left\{S: v_{\mathcal{M}, i}(S)=1\right\}$. So $j$ is a veto player of $\left(N, v_{\mathcal{M}, i}\right)$ and the unit vector $e^{j}$ is a core element of $\left(N, v_{\mathcal{M}, i}\right)$. Hence $\left(N, v_{\mathcal{M}, i}\right)$ is balanced. Since $\left(N, v_{\mathcal{M}, i}\right)$ is balanced for every $i \in N$, any nonnegative combination of the games $\left(N, v_{\mathcal{M}, i}\right), i \in N$, is balanced as well. Therefore $\left(N, v_{\mathcal{M}, w}\right)$ is balanced for every $w \in \mathbb{R}_{+}^{N}$.

" $\Leftarrow$ " Suppose $\left(N, v_{\mathcal{M}, w}\right)$ is balanced for every $w \in \mathbb{R}_{+}^{N}$. This implies that $\left(N, v_{\mathcal{M}, i}\right)$ is balanced for every $i \in N$. Let $i \in N$. If $v_{\mathcal{M}, i}(N)=0$, then the simple balanced game $\left(N, v_{\mathcal{M}, i}\right)$ must be the zero game. In other words, $i \notin \mathcal{M}(S)$ for every $S \in 2^{N}$. Now assume that $v_{\mathcal{M}, i}(N)=1$, i.e. $i \in \mathcal{M}(N)$. Suppose that $\cap\{S: i \in \mathcal{M}(S)\}=\emptyset$. Then $\cup\{N \backslash S: i \in \mathcal{M}(S)\}=N$. Let $x \in \mathbb{R}^{N}$ be a core element of $\left(N, v_{\mathcal{M}, i}\right)$. Then $\sum_{j \in N} x_{j}=v_{\mathcal{M}, i}(N)=1$ and $x_{j} \geq v_{\mathcal{M}, i}(\{j\}) \geq 0$ for every $j \in N$. Let $j \in N$. There is a coalition $S \in 2^{N}$ such that $i \in \mathcal{M}(S)$ and $j \in N \backslash S$. Since $\sum_{k \in S} x_{k} \geq v_{\mathcal{M}, i}(S)=1$ we get $\sum_{k \in N \backslash S} x_{k} \leq 0$, so $x_{k}=0$ for every $k \in N \backslash S$. In particular we have $x_{j}=0$. As this is true for every $j \in N$ we have a contradiction. Therefore $\cap\{S: i \in \mathcal{M}(S)\} \neq \emptyset$, hence $\mathcal{M}$ is veto-rich.

It is well known that the class of TU games that admit a PMAS is a proper subset of the class of totally balanced TU games, i.e. there exist totally balanced games (e.g., glove games) that do not admit a PMAS in general [25]. Given a coalitional map $\mathcal{M}$, the next theorem shows that, whenever all GAGs $\left(N, v_{\mathcal{M}, w}\right)$, for all $w \in \mathbb{R}_{+}^{N}$ are totally balanced, then they equivalently admit a PMAS, and this is also equivalent with the fact that $\mathcal{M}$ is veto-rich and monotonic.

Theorem 3.6 The following statements are equivalent:

I) $\mathcal{M}$ is veto-rich and monotonic; 
II) $\left(N, v_{\mathcal{M}, w}\right)$ admits a PMAS for every $w \in \mathbb{R}_{+}^{N}$;

III) $\left(N, v_{\mathcal{M}, w}\right)$ is totally balanced for every $w \in \mathbb{R}_{+}^{N}$.

Proof "(I) $\Rightarrow(\mathrm{II})$ " Suppose $\mathcal{M}$ is veto-rich and monotonic. Let $i \in N$. If $i \notin \mathcal{M}(S)$ for every $S \in 2^{N}$, then $\left(N, v_{\mathcal{M}, i}\right)$ is the zero game, and hence $v_{\mathcal{M}, i}$ admits a PMAS, because the scheme $\left\{x_{S, k}\right\}_{S \in 2^{N} \backslash\{\emptyset\}, k \in S}$ with $x_{S, k}=0$ for every $S \in 2^{N} \backslash\{\emptyset\}$ and $k \in S$ is a PMAS.

If $i \in \mathcal{M}(N)$ and $\cap\{S: i \in \mathcal{M}(S)\} \neq \emptyset$, then $v_{\mathcal{M}, i}(N)=1$, and take $j \in \cap\{S: i \in \mathcal{M}(S)\}=\cap\left\{S: v_{\mathcal{M}, i}(S)=1\right\}$. So $j$ is a veto player in $\left(N, v_{\mathcal{M}, i}\right)$. We are going to show that the scheme $x=\left\{x_{S, k}\right\}_{S \in 2^{N} \backslash\{\emptyset\}, k \in S}$ such that:

- $x_{S, k}=1$, if $k=j$, and $x_{S, k}=0$, otherwise, for any $S$ with $i \in \mathcal{M}(S)$ and $k \in S$,

- $x_{S, k}=0$, for any $S$ with $i \notin \mathcal{M}(S)$ and $k \in S$,

is a PMAS for $v_{\mathcal{M}, i}$. In order to prove it, first notice that for all $S$, we have $\sum_{k \in S} x_{S, k}=1=v_{\mathcal{M}, i}(S)$, if $i \in \mathcal{M}(S)$, and $\sum_{k \in S} x_{S, k}=0=v_{\mathcal{M}, i}(S)$, if $i \notin \mathcal{M}(S)$ (condition (i) in the definition of PMAS, see Section 2).

In order to prove monotonicity of the scheme $x$ (condition (ii) in the definition of PMAS), let $S \subseteq T$ and $k \in S$. If $x_{S, k}=0$, then obviously $x_{S, k} \leq x_{T, k}$. If $x_{S, k}=1$, then $i \in \mathcal{M}(S)$ and $k=j$. By monotonicity of $\mathcal{M}$ we have that $i \in \mathcal{M}(S) \subseteq \mathcal{M}(T)$. So, $i \in \mathcal{M}(T)$ and then $x_{T, k}=x_{T, j}=1$. Again $x_{S, k} \leq x_{T, k}$ for all $k \in S$.

Since $\left(S, v_{\mathcal{M}, i}\right)$ admits a PMAS for every $i \in N$, any nonnegative combination of the games $\left(N, v_{\mathcal{M}, i}\right), i \in N$, admits a PMAS as well, so $\left(N, v_{\mathcal{M}, w}\right)$ has a PMAS for every $w \in \mathbb{R}_{+}^{N}$.

"(II) $\Rightarrow$ (III)" It follows immediately from the fact that if a game admits a PMAS, then it is also totally balanced.

"(III) $\Rightarrow(\mathrm{I}) "$ Suppose $\left(N, v_{\mathcal{M}, w}\right)$ is totally balanced for every $w \in \mathbb{R}_{+}^{N}$. This implies that $\left(N, v_{\mathcal{M}, w}\right)$ is also balanced for every $w \in \mathbb{R}_{+}^{N}$ and hence by Theorem 3.5, $\mathcal{M}$ is veto-rich. Moreover, it is well known that every totally balanced game is superadditive. Then, by Theorem 3.4, it directly follows that $\mathcal{M}$ is also monotonic.

The next theorem shows that supermodularity of a coalitional map is equivalent with the eponymous property for GAGs on the full domain of vectors of nonnegative contributions.

Theorem 3.7 $\mathcal{M}$ is supermodular if and only if $\left(N, v_{\mathcal{M}, w}\right)$ is supermodular for every $w \in \mathbb{R}_{+}^{N}$.

Proof " $\Rightarrow$ " Take $S, T \in 2^{N}$ with $S \subseteq T$. By supermodularity, we have that $\mathcal{M}(S) \cap \mathcal{M}(T)=\mathcal{M}(S \cap T)=\mathcal{M}(S)$, so $\mathcal{M}(S) \subseteq \mathcal{M}(T)$ or, equivalently, $\mathcal{M}$ is monotonic.

Now, for every $S, T \in 2^{N}$ and $w \in \mathbb{R}_{+}^{N}$ we have that

$$
\begin{aligned}
& v_{\mathcal{M}, w}(S)+v_{\mathcal{M}, w}(T) \\
& =\sum_{i \in \mathcal{M}(S)} w_{i}+\sum_{i \in \mathcal{M}(T)} w_{i} \\
& =\sum_{i \in \mathcal{M}(S) \cup \mathcal{M}(T)} w_{i}+\sum_{i \in \mathcal{M}(S) \cap \mathcal{M}(T)} w_{i} \\
& \leq \sum_{i \in \mathcal{M}(S \cup T)} w_{i}+\sum_{i \in \mathcal{M}(S \cap T)} w_{i} \\
& =v_{\mathcal{M}, w}(S \cup T)+v_{\mathcal{M}, w}(S \cap T),
\end{aligned}
$$

where the inequality follows from the fact that, by monotonicity, $\mathcal{M}(S) \cup \mathcal{M}(T) \subseteq \mathcal{M}(S \cup T)$ and, by supermodularity, $\mathcal{M}(S) \cap \mathcal{M}(T)=\mathcal{M}(S \cap T)$. So, $\left(N, v_{\mathcal{M}, w}\right)$ is supermodular for every $w \in \mathbb{R}_{+}^{N}$.

" $\Leftarrow$ " Now, let $v_{\mathcal{M}, w}$ be supermodular for every $w \in \mathbb{R}_{+}^{N}$. Since supermodular games are also totally balanced, by Theorem 3.6, $\mathcal{M}$ is veto-rich and monotonic. Then, by monotonicity, we have that $\mathcal{M}(S \cap T) \subseteq \mathcal{M}(S)$ and $\mathcal{M}(S \cap T) \subseteq \mathcal{M}(T)$. Then,

$$
\mathcal{M}(S \cap T) \subseteq \mathcal{M}(S) \cap \mathcal{M}(T) .
$$

Moreover, let $S, T \in 2^{N}$ and consider a vector $w \in \mathbb{R}_{+}^{N}$ such that $w_{i}=1$, if $i \in \mathcal{M}(S) \cap \mathcal{M}(T)$, and $w_{i}=0$, otherwise. We have that

$$
\begin{aligned}
& 2|\mathcal{M}(S) \cap \mathcal{M}(T)| \\
& =\sum_{i \in \mathcal{M}(S)} w_{i}+\sum_{\mathcal{M}(T)} w_{i} \\
& =v_{\mathcal{M}, w}(S)+v_{\mathcal{M}, w}(T) \\
& \leq v_{\mathcal{M}, w}(S \cup T)+v_{\mathcal{M}, w}(S \cap T) \\
& =\sum_{i \in \mathcal{M}(S \cup T)} w_{i}+\sum_{i \in \mathcal{M}(S \cap T)} w_{i} \\
& =|\mathcal{M}(S) \cap \mathcal{M}(T)|+|\mathcal{M}(S \cap T)|
\end{aligned}
$$


where the last equality follows from the fact that, by monotonicity of $\mathcal{M}, \mathcal{M}(S) \cap \mathcal{M}(T) \subseteq \mathcal{M}(S \cup T)$ together with the fact that, by relation (4), the elements of $\mathcal{M}(S \cap T)$ are also in $\mathcal{M}(S) \cap \mathcal{M}(T)$, while the inequality directly follows from the definition of supermodularity of $v_{\mathcal{M}, w}$. From relation (5) we have that $|\mathcal{M}(S \cap T)| \geq|\mathcal{M}(S) \cap \mathcal{M}(T)|$, so, together with relation (4), we finally obtain $\mathcal{M}(S \cap T)=\mathcal{M}(S) \cap \mathcal{M}(T)$.

We conclude this section showing the logical dependence relations between properties in Definition 3.1 and their possible combinations.

Proposition 3.8 The following statements about coalitional maps hold true:

i) If $\mathcal{M}$ is veto-rich, then it is also proper;

ii) If $\mathcal{M}$ is supermodular, then it is also monotonic, proper and veto-rich;

iii) $\mathcal{M}$ can be monotonic, proper and veto-rich but not supermodular;

iv) $\mathcal{M}$ can be veto-rich, but neither monotonic nor supermodular;

v) $\mathcal{M}$ can be both monotonic and proper, but neither veto-rich nor supermodular;

vi) $\mathcal{M}$ can be monotonic, but neither proper nor veto-rich nor supermodular;

vii) $\mathcal{M}$ can be proper, but neither monotonic nor veto-rich nor supermodular;

viii) $\mathcal{M}$ can be such that it does not satisfy any of the four properties.

\section{Proof}

Proof of statement (i):

Consider a coalitional map that is veto-rich. Suppose that it is not proper. Then it means that there exist two disjoint coalitions $S, T \in 2^{N}$ such $\mathcal{M}(S) \cap \mathcal{M}(T) \neq \emptyset$. Let $i \in \mathcal{M}(S) \cap \mathcal{M}(T)$. We have that $i \in \mathcal{M}(S)$ and $i \in \mathcal{M}(T)$ with $S \cap T=\emptyset$, which yields a contradiction with the fact that $\mathcal{M}(S)$ is veto-rich.

Proof of statement (ii):

As shown in the proof of Theorem 3.7, if a coalitional map $\mathcal{M}$ is supermodular, then for any $S, T \in 2^{N}$ with $S \subseteq T$ we have that $\mathcal{M}(S) \cap \mathcal{M}(T)=\mathcal{M}(S \cap T)=\mathcal{M}(S)$, so $\mathcal{M}(S) \subseteq \mathcal{M}(T)$ which means that $\mathcal{M}$ is monotonic too. Moreover, taking two disjoint coalitions $S$ and $T$, by the definition of supermodular coalitional map it immediately follows that $\mathcal{M}$ is also proper. Now, let $i \in N$. If there exists $S \in 2^{N}$ such that $i \in \mathcal{M}(S)$, then by monotonicity of the coalitional map $\mathcal{M}$ we have that $i \in \mathcal{M}(N)$. Moreover, by a repeated application of the supermodularity condition, we have that

$$
\emptyset \neq \bigcap_{S \in 2^{N}: i \in \mathcal{M}(S)} \mathcal{M}(S)=\mathcal{M}\left(\bigcap_{S \in 2^{N}: i \in \mathcal{M}(S)} S\right) .
$$

So, by definition of coalitional map, $\bigcap_{S \in 2^{N}: i \in \mathcal{M}(S)} S$ is nonempty, which means that $\mathcal{M}(S)$ is veto-rich too.

For instance, it is easy to check that the identity coalitional map $\mathcal{M}^{2}$ such that $\mathcal{M}^{2}(S)=S$ for all $S \in 2^{N}$ is monotonic, proper, veto-rich and supermodular.

Proof of statement (iii):

Let $i, j, k \in N$ be three distinct elements and consider a coalitional map $\mathcal{M}^{3}$ such that $\mathcal{M}^{3}(N \backslash\{k\})=\mathcal{M}^{3}(N \backslash\{j\})=$ $N \backslash\{j, k\}, \mathcal{M}^{3}(N \backslash\{i\})=N \backslash\{i, k\}, \mathcal{M}^{3}(N)=N \backslash\{k\}$ and $\mathcal{M}^{3}(S)=\emptyset$ for all the remaining coalitions $S \in 2^{N}$. It is easy to check that $\mathcal{M}^{3}$ is monotonic, proper and veto-rich. Now, let $S=N \backslash\{k\}$ and $T=N \backslash\{j\}$. Then $i \in \mathcal{M}^{3}(S) \cap \mathcal{M}^{3}(T)$. However, $\mathcal{M}^{3}(S \cap T)=\mathcal{M}^{3}(N \backslash\{j, k\})=\emptyset$. So $\mathcal{M}^{3}$ is not supermodular.

Proof of statement (iv):

Let $i, j, k \in N$ be three distinct elements and consider a coalitional map $\mathcal{M}^{4}$ such that $\mathcal{M}^{4}(N \backslash\{j, k\})=N \backslash\{j\}$, $\mathcal{M}^{4}(N \backslash\{j\})=N \backslash\{i\}, \mathcal{M}^{4}(N)=N$ and $\mathcal{M}^{4}(S)=\emptyset$ for all the remaining coalitions $S \in 2^{N}$. $\mathcal{M}^{4}$ is veto-rich, as $i \in \mathcal{M}^{4}(N) \cap \mathcal{M}^{4}(N \backslash\{j, k\})$ and $N \cap(N \backslash\{j, k\}) \neq \emptyset ; j \in \mathcal{M}^{4}(N) \cap \mathcal{M}^{4}(N \backslash\{j\})$ and $N \cap(N \backslash\{j\}) \neq \emptyset ;$ and $l \in \mathcal{M}^{4}(N) \cap \mathcal{M}^{4}(N \backslash\{j, k\}) \cap \mathcal{M}^{4}(N \backslash\{j\})$ and $N \cap(N \backslash\{j, k\}) \cap(N \backslash\{j\}) \neq \emptyset$ for all $l \in N \backslash\{i, j\}$. Of course $\mathcal{M}^{4}$ is not monotonic, since $\mathcal{M}^{4}(N \backslash\{j, k\})=N \backslash\{j\}$ but $\mathcal{M}^{4}(N \backslash\{k\})=\emptyset$, and it is not supermodular, since $\mathcal{M}^{4}(N \backslash\{j\}) \cap \mathcal{M}^{4}(N \backslash\{j, k\})=N \backslash\{i, j\} \neq N \backslash\{j\}=\mathcal{M}^{4}((N \backslash\{j\}) \cap(N \backslash\{j, k\}))$. 
Proof of statement (v):

Let $N$ be such that $|N| \geq 3$ and $i \in N$, and consider a coalitional map $\mathcal{M}^{5}$ such that $\mathcal{M}^{5}(N)=\mathcal{M}^{5}(N \backslash\{j\})=\{i\}$ for all $j \in N$ and $\mathcal{M}^{5}(S)=\emptyset$ for all the remaining coalitions $S \in 2^{N}$. It is immediate to check that $\mathcal{M}^{5}$ is both monotonic and proper. On the other hand, $i \in \mathcal{M}^{5}(N)$ but $\bigcap_{j \in N} N \backslash\{j\}=\emptyset$ so $\mathcal{M}^{5}$ is not veto-rich. Of course $\mathcal{M}^{5}$ is not supermodular: for any $j, k \in N$ with $j \neq k$, we have $\mathcal{M}^{5}(N \backslash\{j\}) \cap \mathcal{M}^{5}(N \backslash\{k\})=\{i\} \neq \emptyset=\mathcal{M}^{5}((N \backslash\{j\}) \cap(N \backslash\{k\}))$. Proof of statement (vi):

Let $i \in N$ and consider a coalitional map $\mathcal{M}^{6}$ such that $\mathcal{M}^{6}(S)=\{i\}$ if $i \in S, \mathcal{M}^{6}(N \backslash\{i\})=\{i\}$ and $\mathcal{M}^{6}(T)=\emptyset$ for all the remaining coalitions $T \in 2^{N} . \mathcal{M}^{6}$ is obviously monotonic. But it is neither proper $\left(\right.$ since $\mathcal{M}^{6}(N \backslash\{i\}) \cap$ $\left.\mathcal{M}^{6}(\{i\})=\{i\}\right)$, nor veto-rich (since $\cap\left\{S: i \in \mathcal{M}^{6}(S)\right\}=\emptyset$ ), nor supermodular (since $\mathcal{M}^{6}(N \backslash\{i\}) \cap \mathcal{M}^{6}(\{i\})=\{i\}$ but $\left.\mathcal{M}^{6}((N \backslash\{i\}) \cap\{i\})=\emptyset\right)$.

Proof of statement (vii):

Let $i, j, k \in N$ be three distinct elements and consider a coalitional map $\mathcal{M}^{7}$ such that $\mathcal{M}^{7}(N \backslash\{k\})=N \backslash\{j, k\}$, $\mathcal{M}^{7}(N)=N \backslash\{i\}$ and $\mathcal{M}^{7}(S)=\emptyset$ for all the remaining coalitions $S \in 2^{N} . \mathcal{M}^{7}$ is proper, since $\mathcal{M}^{7}(S) \cap \mathcal{M}^{7}(T)=\emptyset$ for all disjoint coalitions $S$ and $T$. On the other hand, $\mathcal{M}^{7}$ is not monotonic, since $i \in \mathcal{M}^{7}(N \backslash\{k\})$ but $i \notin \mathcal{M}^{7}(N)$ and, for the same reason, it is not even veto-rich. In addition, $\mathcal{M}^{7}$ is not supermodular, since $\mathcal{M}^{7}(N) \cap \mathcal{M}^{7}(N \backslash\{k\})=$ $N \backslash\{i, j, k\} \neq N \backslash\{j, k\}=\mathcal{M}^{7}(N \cap(N \backslash\{k\}))$.

Proof of statement (viii):

Let $i, j \in N$ be two distinct elements and consider a coalitional map $\mathcal{M}^{8}$ such that $\mathcal{M}^{8}(\{i\})=\mathcal{M}^{8}(\{j\})=\{i\}$ and $\mathcal{M}^{8}(S)=\emptyset$ for all the other coalitions $S \in 2^{N}$. It is easy to check that $\mathcal{M}^{8}$ is not proper (we have $\{i\} \cap\{j\}=\emptyset$ but $\left.\mathcal{M}^{8}(\{i\}) \cap \mathcal{M}^{8}(\{j\}) \neq \emptyset\right)$; it is not monotonic $\left(\mathcal{M}^{8}(\{j\})=\{i\} \nsubseteq \emptyset=\mathcal{M}^{8}(\{i, j\})\right)$; it is not veto-rich $\left(i \in \mathcal{M}^{8}(\{i\})\right.$, but $\left.i \notin \mathcal{M}^{8}(N)\right)$; and it is not supermodular $\left(\mathcal{M}^{8}(\{i\}) \cap \mathcal{M}^{8}(\{j\})=\{i\} \neq \emptyset=\mathcal{M}^{8}(\{i\} \cap\{j\})\right)$.

In view of the results provided in this section, we can resume the inclusion relations among families of coalitional maps $\mathcal{M}$ and classes of GAGs $\mathcal{G}^{\mathcal{M}}$ as shown in Figure 1 (recall that given a coalitional map $\mathcal{M}$ on $2^{N}$, the class $\mathcal{G}^{\mathcal{M}}$ is defined as the set of all GAGs $\left(N, v_{\mathcal{M}, w}\right)$ for every $\left.w \in \mathbb{R}_{+}^{N}\right)$.

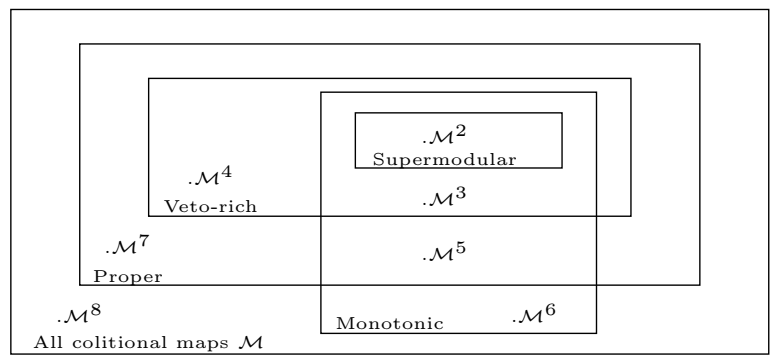

(a)

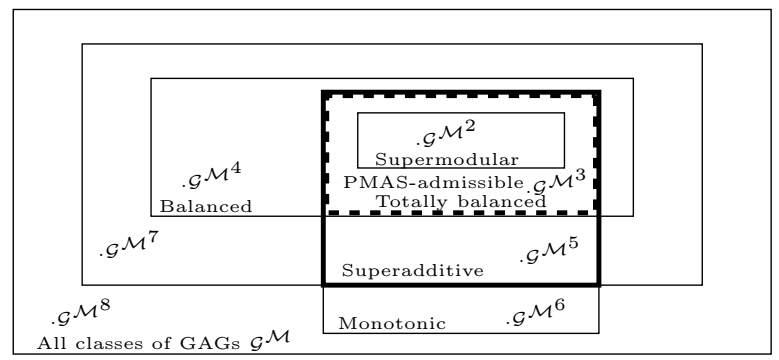

(b)

Figure 1: (a) Euler diagram of sets of coalitonal maps $\mathcal{M}$ characterized by different properties (specified by contours' labels); the elements $\mathcal{M}^{2}, \ldots, \mathcal{M}^{8}$ depicted within the zones, refer to the specific coalitional maps considered in the proof of Proposition 3.8, statements (ii), ..., (viii), respectively. (b) Euler diagram of sets of classes of GAGs $\mathcal{G}^{\mathcal{M}}$ corresponding to zones of coalitional maps represented in the diagram of Figure 1.(a). Notice that, in view of Theorem 3.6, the set of classes of GAGs that are totally balanced coincides with the set of classes of GAGs that admit a PMAS (dashed contour).

\section{Analysis of special classes of GAGs from the literature}

In this section, we illustrate some consequences of the characterizations provided in the previous section on some well known classes of TU games. As we mentioned earlier, all of these classes, with the only exception of glove games, are cost games. For such cost games, we will make use of the corresponding cost saving and dual games, which are interpreted as profit games. 
First, note that all the definitions provided in Section 2 apply to TU games, where $v(S)$, for any coalition $S \in 2^{N}$, represents a profit, whereas some of the inequality signs should be reversed when $v(S)$ represents a cost. So reversing the inequalities in Definitions 2.1.2 and 2.1.3, we obtain, respectively, the conditions of subadditivity and submodularity (or concavity) for game $v$ and, reversing the inequalities in the definition of core and PMAS previously introduced, we obtain the corresponding definitions of core and PMAS for cost games.

Given a cost game $(N, c)$, with cost function $c: 2^{N} \rightarrow \mathbb{R}$, one can also consider the corresponding cost saving game $\left(N, v^{c}\right)$ such that

$$
v^{c}(S)=\sum_{i \in S} c(\{i\})-c(S)
$$

for each coalition $S \in 2^{N}$, where the difference $v^{c}(S)$ between the total cost in the situation where all members of $S$ work alone and the cost in the situation where all members of $S$ cooperate is interpreted as a profit of coalition $S$. In alternative, one can also define the corresponding dual game $\left(N, c^{*}\right)$ such that

$$
c^{*}(S)=c(N)-c(N \backslash S)
$$

for each coalition $S \in 2^{N}$, where the rest $c^{*}(S)$ obtained from the cost of the grand coalition $N$ after the complement of coalition $S$ pays its entire cost in the original game is also interpreted as a profit of coalition $S$.

We now introduce some useful properties for cost games and the corresponding cost saving games.

Proposition 4.1 Let $(N, c)$ be a cost game and let $\left(N, v^{c}\right)$ be the corresponding cost saving game. Then the following statements hold true:

(i) c is submodular iff $v^{c}$ is supermodular;

(ii) $c$ is subadditive iff $v^{c}$ is superadditive;

(iii) $c$ is (totally) balanced iff $v^{c}$ is (totally) balanced;

(iv) c admits a PMAS iff $v^{c}$ admits a PMAS.

Proof Proof of statement (i):

We have that for all $S, T \in 2^{N}$

$$
\begin{array}{ll}
v^{c}(S)+v^{c}(T) \leq v^{c}(S \cup T)+v^{c}(S \cap T) & \Leftrightarrow \\
\sum_{i \in S} c(\{i\})-c(S)+\sum_{i \in T} c(\{i\})-c(T) & \\
\leq \sum_{i \in S \cup T} c(\{i\})-c(S \cup T)+\sum_{i \in S \cap T} c(\{i\})-c(S \cap T) & \Leftrightarrow \\
c(S)+c(T) \geq c(S \cup T)+c(S \cap T), &
\end{array}
$$

where the second equivalence relation follows from the fact that $\sum_{i \in S} c(\{i\})+\sum_{i \in T} c(\{i\})=\sum_{i \in S \cap T} c(\{i\})+$ $\sum_{i \in S \cup T} c(\{i\})$, and where the first and the last inequality are the definition of supermodularity for $v^{c}$ and of submodularity for $c$, respectively.

Proof of statement (ii):

It follows from statement (i), considering coalitions $S, T \in 2^{N}$ such that $S \cap T=\emptyset$.

Proof of statement (iii):

Consider the allocations $x, y \in \mathbb{R}^{N}$ such that $y_{i}=c(\{i\})-x_{i}$ for each $i \in N$. We have that

$$
\begin{array}{ll}
\sum_{i \in N} y_{i}=v^{c}(N)=\sum_{i \in N} c(\{i\})-c(N) & \Leftrightarrow \\
\sum_{i \in N}\left(c(\{i\})-x_{i}\right)=\sum_{i \in N} c(\{i\})-c(N) & \Leftrightarrow \\
\sum_{i \in N} x_{i}=c(N), &
\end{array}
$$

and

$$
\begin{array}{ll}
\sum_{i \in S} y_{i} \geq v^{c}(S)=\sum_{i \in S} c(\{i\})-c(S) & \Leftrightarrow \\
\sum_{i \in S}\left(c(\{i\})-x_{i}\right) \geq \sum_{i \in S} c(\{i\})-c(S) & \Leftrightarrow \\
\sum_{i \in S} x_{i} \leq c(S), &
\end{array}
$$

for each $S \in 2^{N}$ with $S \neq \emptyset$. So, by relations (7) and (8), we have proved that $x$ is efficient and stable for $c$ (equivalently, $c$ is balanced) iff $y$ is efficient and stable for $v^{c}$ (equivalently, $v^{c}$ is balanced). 
Moreover, for each $S \in 2^{N}, S \neq \emptyset$, we have $v_{\mid S}^{c}=v^{c \mid S}$, i.e. the subgame of $v^{c}$ restricted to $S$ coincides with the cost saving game $v^{c_{\mid S}}$ corresponding to $c_{\mid S}$ (which is the subgame of $c$ restricted to $S$ ). Then, again from relations (7) and (8), we have that $c_{\mid S}$ is balanced iff $v^{c_{\mid S}}$ is balanced, for each $S \in 2^{N}, S \neq \emptyset$ (equivalently, $c$ is totally balanced iff $v^{c}$ is totally balanced).

Proof of statement (iv):

Consider the schemes $\left\{x_{S, i}\right\}_{S \in 2^{N} \backslash\{\emptyset\}, i \in S}$ and $\left\{y_{S, i}\right\}_{S \in 2^{N} \backslash\{\emptyset\}, i \in S}$ such that $y_{S, i}=c(\{i\})-x_{S, i}$ for all $S \in 2^{N} \backslash\{\emptyset\}$ and $i \in S$. Similar to relation (7), it is easy to verify that $\sum_{i \in S} x_{S, i}=c(S)$ iff $\sum_{i \in S} y_{S, i}=v^{c}(S)$ for all $S \in 2^{N} \backslash\{\emptyset\}$. To prove the monotonicity condition for the definition of PMAS, notice that

$$
\begin{array}{ll}
y_{S, i} \leq y_{T, i} & \Leftrightarrow \\
c(\{i\})-x_{S, i} \leq c(\{i\})-x_{T, i} & \Leftrightarrow \\
x_{S, i} \geq x_{T, i}, &
\end{array}
$$

for all $S, T \in 2^{N} \backslash\{\emptyset\}$ and $i \in N$ with $i \in S \subset T$. So, we have proved that $\left\{x_{S, i}\right\}_{S \in 2^{N} \backslash\{\emptyset\}, i \in S}$ is a PMAS of $c$ iff $\left\{y_{S, i}\right\}_{S \in 2^{N} \backslash\{\emptyset\}, i \in S}$ is a PMAS of $v^{c}$.

Well-known results for dual games are stated in the following proposition.

Proposition 4.2 Let $(N, c)$ be a cost (profit) game and let its dual $\left(N, c^{*}\right)$ be a profit (cost) game. Then the following statements hold true:

i) $c$ is monotonic iff $c^{*}$ is monotonic;

ii) $C(c)=C\left(c^{*}\right)$ (c and $c^{*}$ have the same core);

iii) $c$ is submodular iff $c^{*}$ is supermodular.

Proof A proof of these statements can be found in the literature, for instance, in the book [3].

In the following, we analyse several classes of TU games from the literature by means of the results introduced in Section 3. Each of the following sections is devoted to a different class of TU games: weighted glove games (Section 4.1), generalized airport games (Section 4.2), fixed tree games (Section 4.3), link connection games (Section 4.4), simple MCST games (Section 4.5), (weighted) coloring games (Section 4.6). Section 4.1 is the only one dealing with a revenue game.

Precisely, the roadmap of each remaining section is as follows. We start with a description of the class of TU games to which the section is devoted, and some related results from the literature. Then, we provide a representation of such TU games as GAGs. We continue showing some consequences of the characterizations introduced in Section 3. To do this, in each section, we first show that the considered coalitional map is monotonic, proper and veto-rich and, for a special case of the coalitional map defined in Section 4.1 and the one in Section 4.3, also supermodular. Second, we use Theorems 3.3 - 3.7 to derive some general results for GAGs based on those coalitional maps, and, in particular, for the class of TU games considered in the section (directly on revenue games, as we do in Section 4.1, or via Propositions 4.1 and 4.2 applied to the corresponding cost saving games, or to the dual games, as we do in Sections 4.2-4.6). Finally, in Sections 4.2, 4.3, 4.4 and 4.6, we conclude the analysis of the class of TU games with some results on special cases.

\subsection{Weighted glove games}

Description of the class of weighted glove games

Given a partition $\{L, R\}$ of the set of players $N$ and a weight vector $w \in \mathbb{R}_{+}^{N}$ (each player $i$ in $L$ owns $w_{i}$ left gloves, each player $j$ in $R$ owns $w_{j}$ right ones), we define a weighted glove game as the TU game $(N, v)$ such that $v(S)=\min \left\{\sum_{i \in S \cap L} w_{i}, \sum_{j \in S \cap R} w_{j}\right\}$, representing the profit obtained by members in $S$ selling their pairs of gloves (sold at selling price of 1 ). Note that players are allowed to have a non-integer number of gloves. If $w_{i}=1$ for every $i \in N$ the game is a standard glove game [24]. Standard glove games are known to be totally balanced. Generalizing the proof in an obvious way shows that weighted glove games are totally balanced as well: for every $S \in 2^{N} \backslash\{\emptyset\}$ allocate $w_{i}$ to every $i \in S \cap L$ and 0 to every $j \in S \cap R$ if $\sum_{i \in S \cap L} w_{i} \leq \sum_{j \in S \cap R} w_{j}$, otherwise allocate 0 to every 
$i \in S \cap L$ and $w_{j}$ to every $j \in S \cap R$. (Weighted) glove games do not have to be PMAS-admissible: in [25] it is shown that the standard glove game with two players owning a left glove and two players owning a right glove does not have a PMAS. In particular, weighted glove games do not have to be supermodular. The following proposition provides a characterization of the subclass of supermodular weighted glove games. Supermodularity and PMAS-admissibility of weighted glove games have been studied in [17] in a more general setting.

Proposition 4.3 Let $(N, v)$ be a weighted glove game with positive weight vector $w$ (so $w_{i}>0$ for every $i \in N$ ) and let $\{L, R\}$ be the partition of $N$ in 'left glove' and 'right glove' players. Then $(N, v)$ is supermodular if and only if $L$ contains precisely one player $l^{*}$ and $w_{l^{*}} \geq \sum_{j \in R} w_{j}$ or $R$ contains precisely one player $r^{*}$ and $w_{r^{*}} \geq \sum_{i \in L} w_{i}$.

Proof " $\Leftarrow "$. Suppose $L$ contains precisely one player $l^{*}$ and $w_{l^{*}} \geq \sum_{j \in R} w_{j}$. Let $i \in N$ and $S \subset T \subseteq N \backslash\{i\}$. If $i=l^{*}$, then $v(S \cup\{i\})-v(S)=\sum_{j \in S} w_{j}-0=\sum_{j \in S} w_{j} \leq \sum_{j \in T} w_{j}=\sum_{j \in T} w_{j}-0=v(T \cup\{i\})-v(T)$. Now assume $i \neq l^{*}$. If $l^{*} \notin S$, then $v(S \cup\{i\})-v(S)=0 \leq v(T \cup\{i\})-v(T)$. If $l^{*} \in S(\subset T)$, then $v(S \cup\{i\})-v(S)=w_{i}=v(T \cup\{i\})-v(T)$. In case $R$ contains precisely one player $r^{*}$ and $w_{r^{*}} \geq \sum_{i \in L} w_{i}$ the argument is similar.

" $\Rightarrow$ ". Suppose $(N, v)$ is supermodular. First, assume that both $L$ and $R$ have at least two players. Choose $a, b \in L, a \neq b$, and $c, d \in R, c \neq d$. We have $v(\{a, c, d\})-v(\{a, d\}) \geq v(\{a, c\})-v(\{a\}) \operatorname{so} \min \left\{w_{a}, w_{c}+w_{d}\right\}-$ $\min \left\{w_{a}, w_{d}\right\} \geq \min \left\{w_{a}, w_{c}\right\}-0, \operatorname{so} \min \left\{w_{a}, w_{c}+w_{d}\right\} \geq \min \left\{w_{a}, w_{c}\right\}+\min \left\{w_{a}, w_{d}\right\}$. So $w_{a} \geq \min \left\{w_{a}, w_{c}+w_{d}\right\} \geq$ $\min \left\{w_{a}, w_{c}\right\}+\min \left\{w_{a}, w_{d}\right\}>\min \left\{w_{a}, w_{c}\right\}$. Hence $\min \left\{w_{a}, w_{c}\right\}=w_{c}$. In a similar way we find $\min \left\{w_{a}, w_{d}\right\}=w_{d}$. Now $w_{a} \geq \min \left\{w_{a}, w_{c}+w_{d}\right\} \geq \min \left\{w_{a}, w_{c}\right\}+\min \left\{w_{a}, w_{d}\right\}=w_{c}+w_{d}$. Using a symmetry argument we get $w_{b} \geq w_{c}+w_{d}, w_{c} \geq w_{a}+w_{b}$ and $w_{d} \geq w_{a}+w_{b}$ as well. Hence $w_{a} \geq w_{c}+w_{d}>w_{c} \geq w_{a}+w_{b}>w_{a}$. A contradiction. So $|L|=1$ or $|R|=1$. Without loss of generality assume that $|L|=1$. If $|R|=1$ we are done so assume that $|R| \geq 2$. Let $L=\left\{l^{*}\right\}$ and let $r^{*} \in R$ be such that $w_{r^{*}}=\min _{j \in R} w_{j}$. We have $v(N)-v\left(N \backslash\left\{r^{*}\right\}\right) \geq$ $v\left(\left\{l^{*}, r^{*}\right\}\right)-v\left(\left\{l^{*}\right\}\right)$ so $\min \left\{w_{l^{*}}, \sum_{j \in R} w_{j}\right\}-\min \left\{w_{l^{*}}, \sum_{j \in R \backslash\left\{r^{*}\right\}} w_{j}\right\} \geq \min \left\{w_{l^{*}}, w_{r^{*}}\right\}-0, \operatorname{so} \min \left\{w_{l^{*}}, \sum_{j \in R} w_{j}\right\} \geq$ $\min \left\{w_{l^{*}}, \sum_{j \in R \backslash\left\{r^{*}\right\}} w_{j}\right\}+\min \left\{w_{l^{*}}, w_{r^{*}}\right\}$. Repeating the arguments above we get $w_{l^{*}} \geq \min \left\{w_{l^{*}}, \sum_{j \in R} w_{j}\right\} \geq$ $\min \left\{w_{l^{*}}, \sum_{j \in R \backslash\left\{r^{*}\right\}} w_{j}\right\}+\min \left\{w_{l^{*}}, w_{r^{*}}\right\}>\min \left\{w_{l^{*}}, \sum_{j \in R \backslash\left\{r^{*}\right\}} w_{j}\right\}$. So, $\min \left\{w_{l^{*}}, \sum_{j \in R \backslash\left\{r^{*}\right\}} w_{j}\right\}=\sum_{j \in R \backslash\left\{r^{*}\right\}} w_{j}$. In a similar way we find $\min \left\{w_{l^{*}}, w_{r^{*}}\right\}=w_{r^{*}}$. So $w_{l^{*}} \geq \min \left\{w_{l^{*}}, \sum_{j \in R} w_{j}\right\} \geq \min \left\{w_{l^{*}}, \sum_{j \in R \backslash\left\{r^{*}\right\}} w_{j}\right\}+$ $\min \left\{w_{l^{*}}, w_{r^{*}}\right\}=\sum_{j \in R \backslash\left\{r^{*}\right\}} w_{j}+w_{r^{*}}=\sum_{j \in R} w_{j}$.

Weighted glove games as generalized additive games

As suggested in [9], a weighted glove game $(N, v)$ can be described as a GAG using the coalitional map $\mathcal{M}$ defined by

$$
\mathcal{M}(S)=\left\{\begin{array}{cc}
S \cap L & \text { if } \\
S \cap R & \sum_{i \in S \cap L} w_{i} \leq \sum_{j \in S \cap R} w_{j} \\
\text { otherwise, }
\end{array}\right.
$$

for all $S \in 2^{N}$. Note that this coalitional map actually depends on the weight vector $w$. It is not possible to construct a 'universal' map $\mathcal{M}$ that generates all weighted glove games for all possible weight vectors. Such an $\mathcal{M}$ would be, according to Theorem 3.6, veto-rich and monotonic and hence, according to the same theorem, all weighted glove games would admit a PMAS.

Consequences of the characterizations in Chapter 3 for weighted glove games

The if-part of Proposition 4.3 can be shown by using Theorem 3.7 as well. Suppose $\{L, R\}$ is a partition of the player set $N$ with $|L|=1$ (the case $|R|=1$ can be treated in a similar way). Let $l^{*}$ be the unique element of $L$. Define the coalitional map $\mathcal{M}: 2^{N} \rightarrow 2^{N}$ by $\mathcal{M}(S)=S \cap R$ if $l^{*} \in S$ and $\mathcal{M}(S)=\emptyset$ otherwise. It is straightforward to check that $\mathcal{M}$ is supermodular. So for every $w \in \mathbb{R}_{+}^{N}$ the game $v^{\mathcal{M}, w}$ is supermodular, according to Theorem 3.7. The game $v^{\mathcal{M}, w}$ is the weighted glove game with weight vector $w$ if $w_{l^{*}} \geq \sum_{j \in R} w_{j}$, where $l^{*}$ is the unique element of $L$.

\subsection{Generalized airport games}

Description of the class of generalized airport games

We recall the definition of airport games [15]. 
Definition 4.4 Consider a finite set $N=\{1, \ldots, n\}$ of players and a vector $w \in \mathbb{R}_{+}^{N}$ with $w_{1} \leq w_{2} \leq \cdots \leq w_{n}$. The airport game associated with $N$ and $w$ is the cost game $(N, c)$ defined by

$$
c(S)=w_{j(S)}
$$

for every $S \in 2^{N}$, where $j(S)=\max \{j: j \in S\}$.

The cost game $(N, c)$ defined according to relation (11) considering a vector $w \in \mathbb{R}_{+}^{N}$ such that $w_{i} \leq w_{n}$ for every $i \in\{1, \ldots, n-1\}$, is called generalized airport game, as introduced in [20].

Given an airport game $(N, c)$ associated with $N$ and $w$, the corresponding cost saving game $\left(N, v^{c}\right)$ is given by

$$
v^{c}(S)=\sum_{i \in S} c(\{i\})-c(S)=\sum_{i \in S} w_{i}-w_{j(S)},
$$

for every $S \in 2^{N}$.

Cost saving games (corresponding to generalized airport games) as generalized additive games Note that the cost saving game $\left(N, v^{c}\right)$ in fact is a $\operatorname{GAG}\left(N, v_{\mathcal{M}, w}\right)$ with coalitional map $\mathcal{M}$ defined by

$$
\mathcal{M}(S)=S \backslash\{j(S)\}
$$

for every $S \in 2^{N}$.

Proposition 4.5 Let $\mathcal{M}$ be the coalitional map as defined in (13). Then $\mathcal{M}$ is monotonic, proper and veto-rich.

Proof Let $S, T \in 2^{N}$ with $S \subseteq T$. Let $i \in \mathcal{M}(S)$. Then $i \in S \subseteq T$ and $i<j(S) \leq j(T)$, so $i \in \mathcal{M}(T)$. This shows that $\mathcal{M}$ is monotonic.

Properness of $\mathcal{M}$ follows from the fact that $\mathcal{M}(S) \subseteq S$ for every $S \in 2^{N}$ (we refer to Remark 3.2).

Veto-richness follows from monotonicity and also from the fact that $\mathcal{M}(S) \subseteq S$ for every $S \in 2^{N}:$ if $i \in \mathcal{M}(S)$ for some $S$ we have, using monotonicity, that $i \in \mathcal{M}(N)$ and $i \in \cap\{T: i \in \mathcal{M}(T)\}$.

The following example illustrates the fact that the coalitional map $\mathcal{M}$ as defined in (13) is not supermodular in case $n \geq 3$.

Example 4.6 Let $N=\{1, \ldots, n\}$ with $n \geq 3$. Consider the two-person coalitions $S=\{1, n-1\}$ and $T=\{1, n\}$. Then $\mathcal{M}(S)=\{1\}, \mathcal{M}(T)=\{1\}$ so $1 \in \mathcal{M}(S) \cap \mathcal{M}(T)$. However, $S \cap T=\{1\}$ and $\mathcal{M}(S \cap T)=\emptyset$, so $\mathcal{M}$ is not supermodular.

Consequences of the characterizations in Chapter 3 for generalized airport games

The following proposition is a direct consequence of Theorems 3.3, 3.4, 3.5, 3.6 and Proposition 4.5.

Proposition 4.7 Let $\mathcal{M}$ be the coalitional map as defined in (13). Then $\left(N, v_{\mathcal{M}, w}\right)$ is monotonic, superadditive, (totally) balanced and PMAS-admissible for every $w \in \mathbb{R}_{+}^{N}$.

In case the weight vector $w$ belongs to the cone $K_{1} \subset \mathbb{R}_{+}^{N}$ defined by $K_{1}=\left\{w \in \mathbb{R}_{+}^{N}: w_{1} \leq w_{2} \leq \cdots \leq w_{n}\right\}$ then, as remarked before, $\left(N, v_{\mathcal{M}, w}\right)$ is the cost saving game corresponding to an airport game and hence Proposition 4.7 provides well-known results concerning airport games. On the somewhat larger cone $K_{2}=\left\{w \in \mathbb{R}_{+}^{N}: w_{i} \leq\right.$ $w_{n}$ for every $\left.i \in\{1, \ldots, n-1\}\right\}$ the games $\left(N, v_{\mathcal{M}, w}\right)$ coincide with the generalized airport games.

Proposition 4.8 Cost saving games corresponding to (generalized) airport games are monotonic, superadditive, (totally) balanced and PMAS-admissible.

Proposition 4.9 (Generalized) airport games are subadditive, (totally) balanced and PMAS-admissible.

Proof The proof follows directly from the properties of cost saving games for (generalized) airport games and Proposition 4.1. 
Some results on a particular class of generalized airport games

Airport games are also known to be submodular (equivalently, by Proposition 4.1, the corresponding cost saving games are supermodular). However, we are not able to show this result using Theorem 3.7 as $\mathcal{M}$ is not supermodular in case $n \geq 3$. So, according to Theorem 3.7, we can conclude that there exists some $w \in \mathbb{R}_{+}^{N}$ such that the corresponding generalized additive game $\left(N, v_{\mathcal{M}, w}\right)$ is not supermodular. This observation was already made in [20], where an example of a generalized airport game was given which is not submodular (and hence the corresponding cost saving game is not supermodular). A natural question now is the following: is it possible to formulate conditions on $\mathcal{M}$ such that $\left(N, v_{\mathcal{M}, w}\right)$ is supermodular for every $w \in K_{1}$ ? The answer is yes. Using (1) and the fact that the cone $K_{1}$ is generated by the vectors $(1,1, \ldots, 1,1),(0,1, \ldots, 1,1),(0,0,1, \ldots, 1,1), \ldots,(0, \ldots, 0,1,1)$ and $(0, \ldots, 0,1)$ we get that $\left(N, v_{\mathcal{M}, w}\right)$ is supermodular for every $w \in K_{1}$ if and only if the following condition holds:

$$
\text { the sum } \sum_{i=j}^{n} v_{\mathcal{M}, i} \text { is supermodular for every } j \in\{1, \ldots, n\} \text {. }
$$

In case $\mathcal{M}$ is defined by (13) we get that $v_{\mathcal{M}, n}$ is the zero game and for every $i \in\{1, \ldots, n-1\}$ we have

$$
v_{\mathcal{M}, i}(S)= \begin{cases}1 & \text { if } i \in S \text { and } i \neq j(S) \\ 0 & \text { otherwise. }\end{cases}
$$

One readily verifies that condition (14) is fulfilled here. In fact, it is enough to notice that by relation (15), for each $S \in 2^{N}$ and $j \in\{1, \ldots, n\}, \sum_{i=j}^{n} v_{\mathcal{M}, i}(S)=|(\{j, \ldots, j(S)\} \cap S) \backslash\{j(S)\}|$ (with the usual convention that $\left.|\emptyset|=0\right)$. Then, for each $k \in N$ and all $S, T \in 2^{N}$ with $k \in S \subseteq T$, it immediately follows that

$$
\sum_{i=j}^{n} v_{\mathcal{M}, i}(S \cup\{k\})-\sum_{i=j}^{n} v_{\mathcal{M}, i}(S) \leq \sum_{i=j}^{n} v_{\mathcal{M}, i}(T \cup\{k\})-\sum_{i=j}^{n} v_{\mathcal{M}, i}(T),
$$

where the inequality is tight if and only if $k=j(S)$ and $k \neq j(T)$.

An alternative way to prove submodularity (as well as monotonicity and balancedness) of airport games, is using the coalitional map for dual fixed tree games on a linear graph as discussed at the end of the next section.

\subsection{Fixed tree games}

Description of the class of fixed tree games

A fixed tree situation or maintenance situation [4] is a tuple $(N, \Gamma, t)$, where $N$ is a finite set of players; $\Gamma=\left(N^{\prime}, E\right)$ is a rooted tree with $N^{\prime}=N \cup\{0\}$ and 0 is the root of the tree; $t \in \mathbb{R}_{+}^{E}$ is the cost function.

Since nodes in $N$ are connected to the root 0 by exactly one path, a natural orientation of edges is defined by the partial preorder on the vertices such that $i \succeq j$ for each $i, j \in N^{\prime}$ if and only if the unique path from $i$ to 0 passes through $j$ (with $i \succeq i$ ). For each $i \in N$ we define the set of predecessors of $i$ in $\Gamma$ as the set $P_{i}^{\Gamma}=\{j \in N: i \succeq j\}$ and by $p(i) \in P_{i}^{\Gamma}$ the immediate predecessor of $i$ on the unique path from $i$ to 0 (i.e., $\{i, p(i)\} \in E$ ). A trunk of $\Gamma$ is a set of vertices $T \subseteq N^{\prime}$ which is closed under relation $\succeq$ (i.e., $i \in T$ and $i \succeq j \Rightarrow j \in T$ ). For any coalition $S \subseteq N$, the trunk of $S$, denoted by $T_{S} \subseteq N^{\prime}$, is the smallest trunk containing all nodes $S$.

The fixed tree game, associated with a fixed tree situation $(N, \Gamma, t)$, is the cost game $(N, c)$, where each coalition $S \in 2^{N}$ must support the (minimal) cost of maintaining all paths from players in $S$ to 0 , precisely:

$$
c(S)=\sum_{i \in T_{S} \backslash\{0\}} t_{\{i, p(i)\}}
$$

Given a fixed tree game $(N, c)$ associated with $(N, \Gamma, t)$, the corresponding dual game $\left(N, c^{*}\right)$ is defined as:

$$
c^{*}(S)=c(N)-c(N \backslash S)=\sum_{e \in E} t_{e}-\sum_{i \in\left(T_{N \backslash S}\right) \backslash\{0\}} t_{\{i, p(i)\}},
$$

for every $S \in 2^{N}$. 
Dual games (corresponding to fixed tree games) as generalized additive games

In this case, the dual game $c^{*}$ coincides with the $\operatorname{GAG}\left(N, v_{\mathcal{M}, w}\right)$ with $w_{i}=t_{\{i, p(i)\}}$, and the coalitional map $\mathcal{M}$ is defined as follows:

$$
\mathcal{M}(S)=N \backslash\left(T_{N \backslash S}\right),
$$

for every $S \in 2^{N}$.

Proposition 4.10 Let $\Gamma=\left(N^{\prime}, E\right)$ be a rooted tree and let $\mathcal{M}$ be the coalitional map as defined in (17). Then $\mathcal{M}$ is monotonic, proper, veto-rich and supermodular.

Proof In order to prove that $\mathcal{M}$ is monotonic, consider any two coalitions $U, W \in 2^{N}$ with $U \subseteq W$. Then, $N \backslash U \supseteq N \backslash W$, implying that the trunk of $N \backslash W$ is included in the trunk of $N \backslash U$, i.e. $T_{N \backslash U} \supseteq T_{N \backslash W}$. So, $\mathcal{M}(U)=N \backslash\left(T_{N \backslash U}\right) \subseteq N \backslash\left(T_{N \backslash W}\right)=\mathcal{M}(W)$.

Properness of $\mathcal{M}$ follows from the fact that $\mathcal{M}(S) \subseteq S$ for every $S \in 2^{N}$ (see also Remark 3.2). In fact, let $S \in 2^{N}$ and let $j \notin S$. Then $j \in N \backslash S$, so $j \in T_{N \backslash S}$, and $j \notin N \backslash\left(T_{N \backslash S}\right)=\mathcal{M}(S)$. This means that if $j \in \mathcal{M}(S)$, then $j \in S$, and we have proved that $\mathcal{M}(S) \subseteq S$ for every $S \in 2^{N}$.

Veto-richness follows from monotonicity and also from the fact that $\mathcal{M}(S) \subseteq S$ for every $S \in 2^{N}:$ if $i \in \mathcal{M}(S)$ for some $S$ we have, using monotonicity, that $i \in \mathcal{M}(N)$ and, from the fact that $\mathcal{M}(S) \subseteq S$, that $i \in \cap\{U: i \in \mathcal{M}(U)\}$.

We now prove supermodularity of $\mathcal{M}$. Let $U, W \in 2^{N}$. From the fact that $\mathcal{M}(U \cap W) \subseteq \mathcal{M}(U)$ and $\mathcal{M}(U \cap$ $W) \subseteq \mathcal{M}(W)$ we have immediately that $\mathcal{M}(U \cap W) \subseteq \mathcal{M}(U) \cap \mathcal{M}(W)$. In order to show that it also holds $\mathcal{M}(U) \cap \mathcal{M}(W) \subseteq \mathcal{M}(U \cap W)$, we first notice that if $i \in \overline{\mathcal{M}}(U) \cap \mathcal{M}(W)$, then it means that there is no $j \in N$ with either $j \in N \backslash U$ or $j \in N \backslash W$ such that $i \in P_{j}^{\Gamma}$. Equivalently, there is no $j \in N \backslash(U \cap W)$ such that $i \in P_{j}^{\Gamma}$. Then, it follows that $i \in \mathcal{M}(U \cap W)$ too, which concludes the proof.

Consequences of the characterizations in Chapter 3 for fixed tree games

The following proposition is a direct consequence of Theorems 3.3, 3.4, 3.5, 3.6, 3.7 and Proposition 4.10.

Proposition 4.11 Let $\Gamma=\left(N^{\prime}, E\right)$ be a rooted tree and let $\mathcal{M}$ be the coalitional map as defined in (17). Then $\left(N, v_{\mathcal{M}, w}\right)$ is monotonic, superadditive, (totally) balanced, PMAS-admissible and supermodular for every $w \in \mathbb{R}_{+}^{E}$.

As a direct consequence of relations (16) and (17) we can state the following.

Proposition 4.12 Dual games corresponding to fixed tree games are monotonic, superadditive, (totally) balanced, PMAS-admissible and supermodular.

Proposition 4.13 Fixed tree games are monotonic, balanced and submodular.

Proof The proof follows directly from the properties of dual games of fixed tree games and Proposition 4.2.

Remark 4.14 As we already noticed, a submodular game is also totally balanced and PMAS-admissible. However, in Proposition 4.13, we only show the direct consequences of Proposition 4.2 on dual games of fixed tree games.

Some results on a particular class of fixed tree games

Notice that if $\left(N^{\prime}, E\right)$ is a linear graph, then $(N, c)$ is an airport game. Then, by Proposition 4.13 , it immediately follows that also airport games are monotonic, balanced and submodular.

\subsection{Link connection games}

Description of the class of link connection games

We recall the definition of link connection games, as introduced in [16] and, as a special case of a broader class of games on matroids in [19]. Let $(V, E)$ be an undirected graph with vertex set $V$ and edge set $E$ and let $w \in \mathbb{R}_{+}^{E}$. The link connection game associated with $(V, E)$ and $w$ is the cost game $(E, c)$, where the set of edges is the set of players, and the cost of a coalition $S \in 2^{E}$ is the minimal cost of a set of edges $T \subseteq S$ such that $(V, T)$ and $(V, S)$ have the same connected components. 
Definition 4.15 Let $(V, E)$ be an undirected graph and let $w \in \mathbb{R}_{+}^{E}$. The link connection game associated with $(V, E)$ and $w$ is the cost game $(E, c)$, such that

$$
c(S)=\min \left\{W(T) \mid T \subseteq S \text { and } \mathcal{P}_{T}=\mathcal{P}_{S}\right\}
$$

for every $S \subseteq E$, where $W(T)=\sum_{e \in T} w_{e}$ and $\mathcal{P}_{T}$ denotes the set of all connected components in graph $\left(V_{S}, T\right)$ for any $T \subseteq S$ (here, $V_{S}$ is the set of all nodes of edges in $S$ ).

In other words, $c(S)$ is the cost of a graph of minimum cost $\left(V_{S}, T\right), T \subseteq S$, such that the set of connected components in $\left(V_{S}, T\right)$ coincides with the set of connected components in $\left(V_{S}, E_{S}\right)$. Given a link connection game $(E, c)$ associated with $(V, E)$ and $w$, the corresponding cost saving game $\left(E, v^{c}\right)$ is defined as follows:

$$
v^{c}(S)=\sum_{e \in S} w_{e}-c(S)
$$

for every $S \subseteq E$.

Cost saving games (corresponding to link connection games) as generalized additive games

We will show that the cost saving game $\left(E, v^{c}\right)$ is in fact also a GAG $\left(N, v_{\mathcal{M}, w}\right)$. First, we construct the coalitional map $\mathcal{M}: 2^{E} \rightarrow 2^{E}$ for some undirected graph $(V, E)$. We start with listing the edges in $E$ in some order $E=\left\{e_{1}, e_{2}, e_{3}, \ldots, e_{|E|}\right\}$ (not yet having a cost vector $w \in \mathbb{R}_{+}^{E}$ in mind). For every $S \subseteq E$ an edge $e_{k} \in S$, $(k \in\{1, \ldots,|E|\})$ is called superfluous in $S$ if it forms a cycle with its predecessors in $S$, more precisely, if $\left(V,\left\{e_{1}, \ldots, e_{k-1}\right\} \cap S\right)$ and $\left(V,\left\{e_{1}, \ldots, e_{k}\right\} \cap S\right)$ have the same connected components. Now $\mathcal{M}$ selects the collection of superfluous edges in any coalition:

$$
\mathcal{M}(S)=\{e \in S: e \text { is superfluous in } S\}
$$

for every $S \subseteq E$.

Proposition 4.16 Let $\mathcal{M}$ be the coalitional map as defined in (20). Then $\mathcal{M}$ is monotonic, proper and veto-rich.

Proof Let $S, T \in 2^{E}$ with $S \subseteq T$. Let $e \in \mathcal{M}(S)$. Then $e$ forms a cycle with his predecessors in $S$, so definitely also a cycle with his predecessors in $T$. Therefore $e \in \mathcal{M}(T)$ which shows that $\mathcal{M}$ is monotonic.

Properness of $\mathcal{M}$ follows from the fact that $\mathcal{M}(S) \subseteq S$ for every $S \in 2^{E}$ (see Remark 3.2).

Veto-richness follows from monotonicity and also from the fact that $\mathcal{M}(S) \subseteq S$ for every $S \in 2^{E}$ : if $e \in \mathcal{M}(S)$ for some $S$ we have, using monotonicity, that $e \in \mathcal{M}(N)$ and $e \in \cap\{T: e \in \mathcal{M}(T)\}$.

In the following example we will show that it is possible that the coalitional map $\mathcal{M}$ as defined in (20) is supermodular, but that this is not necessarily true.

Example 4.17 Consider the graph $\left(V_{1}, E_{1}\right)$ with $V_{1}=\{1,2,3,4\}$ and $E_{1}=\{12,14,23,34\}$ (in order to avoid cumbersome notations, we write ij instead of $\{i, j\})$. Choose the ordering of the edges in $E_{1}$ such that $e_{1}=12$, $e_{2}=23, e_{3}=34$ and $e_{4}=14$. Now the corresponding coalitional map $\mathcal{M}_{1}$ is such that $\mathcal{M}_{1}\left(E_{1}\right)=\left\{e_{4}\right\}$ and $\mathcal{M}_{1}(S)=\emptyset$ for all $S \subseteq E_{1}, S \neq E_{1}$. It is straightforward to see that $\mathcal{M}_{1}$ is supermodular.

Now consider the graph $\left(V_{2}, E_{2}\right)$ with $V_{2}=\{1,2,3,4\}$ and $E_{2}=\{12,13,14,23,34\}$. Choose the ordering of the edges in $E_{1}$ such that $e_{1}=12, e_{2}=23, e_{3}=34, e_{4}=14$ and $e_{5}=13$. Now the corresponding coalitional map $\mathcal{M}_{2}$ is such that $\mathcal{M}_{2}\left(E_{2}\right)=\left\{e_{4}, e_{5}\right\}, \mathcal{M}_{2}\left(\left\{e_{1}, e_{2}, e_{5}\right\}\right)=\mathcal{M}_{2}\left(\left\{e_{3}, e_{4}, e_{5}\right\}\right)=\mathcal{M}_{2}\left(\left\{e_{1}, e_{2}, e_{3}, e_{5}\right\}\right)=\mathcal{M}_{2}\left(\left\{e_{1}, e_{2}, e_{4}, e_{5}\right\}\right)=$ $\mathcal{M}_{2}\left(\left\{e_{1}, e_{3}, e_{4}, e_{5}\right\}\right)=\mathcal{M}_{2}\left(\left\{e_{2}, e_{3}, e_{4}, e_{5}\right\}\right)=\left\{e_{5}\right\}, \mathcal{M}_{2}\left(\left\{e_{1}, e_{2}, e_{3}, e_{4}\right\}\right)=\left\{e_{4}\right\}$ and $\mathcal{M}_{2}(S)=\emptyset$ for all other $S$. Now with $S=\left\{e_{3}, e_{4}, e_{5}\right\}$ and $T=\left\{e_{1}, e_{2}, e_{5}\right\}$ we have $\mathcal{M}_{2}(S \cap T)=\mathcal{M}_{2}\left(\left\{e_{5}\right\}\right)=\emptyset$, whereas $\mathcal{M}_{2}(S) \cap \mathcal{M}_{2}(T)=\left\{e_{5}\right\}$. So $\mathcal{M}_{2}$ is not supermodular.

Now, we will show that the cost saving games $\left(E, v^{c}\right)$ as defined in (19) and corresponding to link connection games as defined in (18), form a subset of GAGs $\left(E, v_{\mathcal{M}, w}\right)$ with $\mathcal{M}$ specified by $(20)$. Let $(E, c)$ be the link connection game associated with undirected graph $(V, E)$ and cost vector $w \in \mathbb{R}_{+}^{E}$. Choose the ordering of the edges in $E$ according to increasing costs, i.e. $E=\left\{e_{1}, e_{2}, e_{3}, \ldots, e_{|E|}\right\}$ such that $w_{e_{1}} \leq w_{e_{2}} \leq \cdots \leq w_{e_{|E|}}$, and let $\mathcal{M}$ be the 
corresponding coalitional map. Let $S \subseteq E$. The graph $(V, S)$ partitions the vertex set $V$ into components. Some components may be singletons, some may be trees and the other components are connected components containing cycles. In order to find a subset $T \subseteq S$ of minimal cost that results in the same partition of $V$ into components we can use the well-known algorithm of Prim: reduce any component in $(V, S)$ with a cycle to a tree by removing edges that form a cycle with the cheaper edges in $S$. Since we have chosen the order of $E$ with respect to increasing costs this process boils down to removing the superfluous edges in $S$, i.e. removing the edges in $\mathcal{M}(S)$. So an optimal network for coalition $S$ is $(V, S \backslash \mathcal{M}(S))$ and the cost saving, going from $(V, S)$ to $(V, S \backslash \mathcal{M}(S))$, is equal to $\sum_{e \in \mathcal{M}(S)} w_{e}=v_{\mathcal{M}, w}(S)$. As this is true for every $S \subseteq E$ we get $\left(E, v^{c}\right)=\left(E, v_{\mathcal{M}, w}\right)$.

Consequences of the characterizations in Chapter 3 for link connection games

The following proposition is a direct consequence of Theorems 3.3, 3.4, 3.5, 3.6 and Proposition 4.16.

Proposition 4.18 Let $(V, E)$ be an undirected graph and $\mathcal{M}$ the coalitional map as defined in (20). Then $\left(E, v_{\mathcal{M}, w)}\right.$ is monotonic, superadditive, (totally) balanced and PMAS-admissible for every $w \in \mathbb{R}_{+}^{E}$.

As a consequence of Proposition 4.18 we obtain the following one.

Proposition 4.19 Cost saving games corresponding to link connection games are monotonic, superadditive, (totally) balanced and PMAS-admissible.

Proposition 4.20 Link connection games are subadditive, (totally) balanced and PMAS-admissible.

Proof The proof follows directly from the properties of cost saving games corresponding to link connection games and by Proposition 4.1.

Some results on a particular class of link connection games

Cost saving games corresponding to link connection games are generalized additive games with coalitional map $\mathcal{M}$ specified by (20) and cost vectors $w$ in the cone $K \subset \mathbb{R}_{+}^{E}$ specified by $0 \leq w_{e_{1}} \leq w_{e_{2}} \leq \cdots \leq w_{e_{|E|}}$. This cone is generated by the vectors $(1,1, \ldots, 1,1),(0,1, \ldots, 1,1),(0,0,1, \ldots, 1,1), \ldots,(0, \ldots, 0,1,1)$ and $(0, \ldots, 0,1)$ and this enables us, like in the example of airport games, to formulate necessary and sufficient conditions on $\mathcal{M}$ (and hence on the undirected graph $(V, E))$, for getting supermodular cost saving games for every $w \in K$. We have $\left(E, v_{\mathcal{M}, w}\right)$ is supermodular for every $w \in K$ if and only if the sum

$$
\sum_{i=j}^{|E|} v_{\mathcal{M}, e_{i}}
$$

is supermodular for every $j \in\{1, \ldots,|E|\}$. Here the games $\left(E, v_{\mathcal{M}, e_{i}}\right)$ are monotonic simple games with as minimal winning coalitions the simple cycles in $(V, E)$ that contain $e_{i}$ but no edge with an higher index.

Example 4.21 Reconsider the two undirected graphs $\left(V_{1}, E_{1}\right)$ and $\left(V_{2}, E_{2}\right)$ of Example 4.17. We have $v_{\mathcal{M}_{1}, e_{i}}$ is the zero game for $i \in\{1,2,3\}$ and $v_{\mathcal{M}_{1}, e_{4}}=u_{E_{1}}$. So $\sum_{i=j}^{4} v_{\mathcal{M}, e_{i}}$ is supermodular for every $j \in\{1,2,3,4\}$, hence $\left(E, v_{\mathcal{M}_{1}, w}\right)$ is supermodular for every $w \in K$.

For graph $\left(V_{2}, E_{2}\right)$ we have that $v_{\mathcal{M}_{2}, e_{i}}$ is the zero game for $i \in\{1,2,3\}, v_{\mathcal{M}_{2}, e_{4}}=u_{\left\{e_{1}, e_{2}, e_{3}, e_{4}\right\}}$ and $v_{\mathcal{M}_{2}, e_{5}}=$ $u_{\left\{e_{1}, e_{2}, e_{5}\right\},\left\{e_{3}, e_{4}, e_{5}\right\}}$. As $v_{\mathcal{M}_{2}, e_{5}}$ is not supermodular (it is a monotonic simple game that is not a unanimity game) we conclude that it is not true that $\left(E, v_{\mathcal{M}_{2}, w}\right)$ is supermodular for every $w \in K$.

\subsection{Simple MCST games}

Description of the class of simple MCST games

We recall the definition of minimum cost spanning tree (MCST) game, as introduced in [20, 27]. First, we need to introduce some graph notions specific for this section. Given a finite set $N=\{1, \ldots, n\}$ and a source denoted by 0 , let $\left(N^{\prime}, E_{N^{\prime}}\right)$ be an undirected complete graph with vertex set $N^{\prime}=N \cup\{0\}$ and edge set $E_{N^{\prime}}$ and, let $z \in \mathbb{R}_{+}^{E_{N^{\prime}}}$ be a vector of nonnegative weights on the edges in $E_{N^{\prime}}$. An undirected graph $\left(S^{\prime}, T\right), T \subseteq E_{S^{\prime}}$, is a spanning network 
on $S^{\prime}=S \cup\{0\}, S \in 2^{N}$, if for every $i \in S$ there is a path in $\left(S^{\prime}, T\right)$ from $i$ to the source. For any $S \in 2^{N}$, it is possible to determine at least one spanning tree on $S^{\prime}$ for $z$, i.e. a spanning network $\left(S^{\prime}, T\right)$ (without cycles) on $S^{\prime}$, of minimum cost $z(T)=\sum_{e \in T} z_{e}$, which is called an MCST on $S^{\prime}$ for $z$.

Definition 4.22 The MCST game associated with $\left(N^{\prime}, E_{N^{\prime}}\right)$ and $z$ is the cooperative cost game $\left(N, c_{z}\right)$ defined by

$$
c_{z}(S)=\min \left\{z(T) \mid\left(S^{\prime}, T\right) \text { is a spanning network on } S^{\prime}\right\}
$$

for every $S \in 2^{N}$.

A simple MCST game is an MCST game $\left(N, c_{z}\right)$ such that $z \in\{0,1\}^{E_{N^{\prime}}}$ (i.e., the weights of edges are 0 or 1 ). Now, let $S \in 2^{N} \backslash\{\emptyset\}$. Two nodes $i$ and $j$ in $S^{\prime}$ are $\left(z, S^{\prime}\right)$-connected if there exists a sequence of nodes $i=i_{0}, \ldots, i_{k}=j$ in $S \cup\{0\}$ with $z\left(\left\{i_{s}, i_{s+1}\right\}\right)=0$ for every $s \in\{0, \ldots, k-1\}$. A $\left(z, S^{\prime}\right)$-component of $S \cup\{0\}$ is a maximal subset of $S \cup\{0\}$ with the property that any two nodes in this subset are $\left(z, S^{\prime}\right)$-connected. The number of $\left(z, S^{\prime}\right)$-components is denoted by $n\left(z, S^{\prime}\right)$. Clearly, the collection of $\left(z, S^{\prime}\right)$-components forms a partition of $S^{\prime}$ which is denoted by $\mathcal{W}_{\mid S^{\prime}}$. As shown in $[8,21,27]$ we have that

$$
c_{z}(S)=n\left(z, S^{\prime}\right)-1
$$

for every $S \in 2^{N} \backslash\{\emptyset\}$.

Given a simple MCST game $\left(N, c_{z}\right)$ associated with $\left(N^{\prime}, E_{N^{\prime}}\right)$ and $z \in\{0,1\}^{E_{N^{\prime}}}$, the corresponding cost saving game $\left(N, v^{c_{z}}\right)$ is given by

$$
v^{c_{z}}(S)=\sum_{i \in S} z_{\{i, 0\}}-c_{z}(S)
$$

for every $S \in 2^{N}$.

Cost saving games (corresponding to simple MCST games) as generalized additive games

Note that the cost saving game $\left(N, v^{c_{z}}\right)$ is in fact a $\operatorname{GAG}\left(N, v_{\mathcal{M}, w}\right)$ with $w_{i}=1$ for each $i \in N$ and with coalitional map $\mathcal{M}$ defined by

$$
\mathcal{M}(S)=I_{S} \backslash \bigcup_{T \in \mathcal{W}_{\mid S^{\prime}}: 0 \notin T}\{j(T)\},
$$

where, for every $S \in 2^{N}, I_{S}$ is the set of nodes in $S$ such that $z(\{i, 0\})=1$, and $j(T)=\max \{j: j \in T\}$ for all $T \in \mathcal{W}_{\mid S^{\prime}}$. In fact, it is sufficient to notice that $\sum_{i \in S} z_{\{i, 0\}}=\left|I_{S}\right|$ (by definition of $I_{S}$ ), and that all the elements in the $\left(z, S^{\prime}\right)$-connected components not including 0 belong to $I_{S}$, and then we can obtain $v^{c_{z}}(S)$ just counting the element in $I_{S}$ minus exactly one element of each $\left(z, S^{\prime}\right)$-connected component $T \in \mathcal{W}_{\mid S^{\prime}}$ not containing 0 (for instance, the one with largest index $j(T)$ ).

Proposition 4.23 Let $\mathcal{M}$ be the coalitional map as defined in (24). Then $\mathcal{M}$ is monotonic, proper and veto-rich.

Proof Let $S, T \in 2^{N}$ with $S \subseteq T$. Let $i \in \mathcal{M}(S)$. Then $i \in I_{S} \subseteq S$ and $i \in I_{T} \subseteq T$. This means that there exists $C \in \mathcal{W}_{\mid S^{\prime}}$ such that $i \in C$ and $K \in \mathcal{W}_{\mid T^{\prime}}$ such that $i \in K$. Note that $C \subseteq K$. If $0 \in K$, then $i \in \mathcal{M}(T)$. Now, suppose that $0 \notin K$ (so $0 \notin C)$. Then $i<j(C) \leq j(K)$ and it again follows that $i \in \mathcal{M}(T)$, and we have shown that $\mathcal{M}$ is monotonic.

Properness of $\mathcal{M}$ follows from the fact that $\mathcal{M}(S) \subseteq S$ for every $S \in 2^{N}$.

Veto-richness follows from monotonicity and also from the fact that $\mathcal{M}(S) \subseteq S$ for every $S \in 2^{N}$ : if $i \in \mathcal{M}(S)$ for some $S$ we have, using monotonicity, that $i \in \mathcal{M}(N)$ and $i \in \cap\{T: i \in \mathcal{M}(T)\}$.

Consequences of the characterizations in Chapter 3 for simple MCST games

The following proposition is a direct consequence of Theorems 3.3, 3.4, 3.5, 3.6 and Proposition 4.23.

Proposition 4.24 Let $\left(N^{\prime}, E_{N^{\prime}}\right)$ be an undirected complete graph and let $\mathcal{M}$ be the coalitional map as defined in (24). Then $\left(N, v_{\mathcal{M}, w}\right)$ is monotonic, superadditive, (totally) balanced and PMAS-admissible for every $w \in \mathbb{R}_{+}^{N}$.

Proposition 4.24 holds in particular for the case $w_{i}=1$ for all $i \in N$. Then, by relation (24) we have the following. 
Proposition 4.25 Cost saving games corresponding to simple MCST games are monotonic, superadditive, (totally) balanced and PMAS-admissible.

Proposition 4.26 Simple MCST games are subadditive, (totally) balanced and PMAS-admissible.

Proof The proof follows directly from the properties of cost saving games corresponding to simple MCST games and by Proposition 4.1.

In $[8,21,27]$ it has been shown that every MCST is a nonnegative combination of simple MCST games. Then, by Proposition 4.26 it immediately follows that also MCST games are subadditive, (totally) balanced and PMASadmissible, as already noticed in [21].

\section{6 (Weighted) minimum coloring games}

Description of the class of (weighted) coloring games

Let $\Gamma=(N, E)$ be an undirected graph and $w \in \mathbb{N}^{N}$ a positive integer weight vector. A clique is a set $S \in 2^{N}$ such that $\{i, j\} \in E$ for every $i, j \in S, i \neq j$. The weight of this clique is $\sum_{i \in S} w_{i}$. A clique with maximum weight is called a maximum weighted clique and the corresponding weight the weighted clique number of $G$. This number is denoted by $\omega_{w}(\Gamma)$. For $k \in \mathbb{N}$ a $k$-coloring of graph $\Gamma$ with respect to weight vector $w$ is a function $h$ that assigns a set of $w_{i}$ different colors to every vertex $i \in N$ such that adjacent vertices receive disjoint sets of colors and at most $k$ colors are used. Formally, such a coloring is a map $h: N \rightarrow 2^{\{1, \ldots, k\}}$ such that $|h(i)|=w_{i}$ for every $i \in N$ and $h(i) \cap h(j)=\emptyset$ for every $\{i, j\} \in E$. The minimum number $k$ such that a $k$-coloring of $\Gamma$ with respect to $w$ exists is called the weighted chromatic number of $\Gamma$ with respect to $w$ and denoted as $\chi_{w}(\Gamma)$. The weighted minimum coloring game [12] associates with every coalition the weighted chromatic number of $\Gamma_{\mid S}$ with respect to $w_{S}=\left(w_{i}\right)_{i \in S}$.

Definition 4.27 The weighted minimum coloring game [12] on $\Gamma=(N, E)$ with weight vector $w \in \mathbb{N}^{N}$ is the cost game $\left(N, c^{\Gamma, w}\right)$ defined by

$$
c^{\Gamma, w}(S)=\chi_{w_{S}}\left(\Gamma_{\mid S}\right)
$$

for every $S \in 2^{N}$.

In case $w_{i}=1$ for every $i \in N$ then $\omega_{w}(\Gamma)=\omega(\Gamma)$ and $\chi_{w}(\Gamma)=\chi(\Gamma)$ where $\omega(\Gamma)$ and $\chi(\Gamma)$ are the well known clique and chromatic numbers of $\Gamma$. Moreover $\left(N, c^{\Gamma, w}\right)=\left(N, c^{\Gamma}\right)$, where $\left(N, c^{\Gamma}\right)$ is the minimum coloring game of $\Gamma([10,11])$.

A graph $\Gamma=(N, E)$ is called perfect if $\omega\left(\Gamma_{\mid S}\right)=\chi\left(\Gamma_{\mid S}\right)$ for every $S \in 2^{N}$, i.e. the clique and chromatic numbers are the same for the full graph and every induced subgraph. Perfect graphs are known to be weighted perfect as well (see, for example [23]): for every weight vector the weighted clique and weighted chromatic numbers are the same for the full graph and every induced subgraph. A graph $\Gamma=(N, E)$ is called $\left(2 K_{2}, P_{4}\right)$-free if there is no $S \in 2^{N}$, $|S|=4$ such that $\Gamma_{\mid S}$ is a graph with two edges that have no vertex in common $\left(2 K_{2}\right)$ or $\Gamma_{\mid S}$ is a linear graph with three edges $\left(P_{4}\right)$. A graph $\Gamma=(N, E)$ is called complete multipartite if there is a partition $\left\{P_{1}, P_{2}, \ldots, P_{r}\right\}$ of the vertex set $N$ such that for any two vertices $i \in P_{k}, j \in P_{l}$ we have $\{i, j\} \in E$ if and only if $k \neq l$.

From the literature we know the following results relating properties of graphs to properties of the related (weighted) minimum coloring games: $\Gamma$ is perfect if and only if $c^{\Gamma}$ is totally balanced [10], $\Gamma$ is perfect if and only if $c^{\Gamma, w}$ is totally balanced for every $w \in \mathbb{N}^{N}[12], \Gamma$ is $\left(2 K_{2}, P_{4}\right)$-free if and only if $c^{\Gamma}$ has a PMAS [11], $\Gamma$ is $\left(2 K_{2}, P_{4}\right)$ free if and only if $c^{\Gamma, w}$ has a PMAS for every $w \in \mathbb{N}^{N}[12], \Gamma$ is complete multipartite if and only if $c^{\Gamma}$ is submodular [22] and $\Gamma$ is complete multipartite if and only if $c^{\Gamma, w}$ is submodular for every $w \in \mathbb{N}^{N}[12]$.

Cost saving games (corresponding to (weighted) minimum coloring games) as generalized additive games First, consider the following example.

Example 4.28 Consider the graph $\Gamma=(N, E)$ with $N=\{1,2,3,4\}$ and $E=\{\{1,2\},\{3,4\}\}$ (so $\Gamma$ is isomorphic to $\left.2 K_{2}\right)$ and let $w \in \mathbb{N}^{N}$ be such that $w_{i}=1$ for every $i \in N$. As $\Gamma$ is perfect but not $\left(2 K_{2}, P_{4}\right)$-free we know that $c^{\Gamma}$ is totally balanced but that it does not have a PMAS. Let $v^{c^{\Gamma}}$ be the cost savings game, corresponding to $c^{\Gamma}$. It is readily verified that $v^{c^{\Gamma}}(N)=2, v^{c^{\Gamma}}(S)=0$ if $S \in\{\{1\},\{2\},\{3\},\{4\},\{1,2\},\{3,4\}\}$ and $v^{c^{\Gamma}}(S)=1$ otherwise (in 
fact $v^{c^{\Gamma}}$ is a glove game with two left and two right gloves). With $\mathcal{M}$ defined by $\mathcal{M}(N)=\{1,2\}, \mathcal{M}(S)=\{1\}$ if $S \in\{\{1,3\},\{1,4\},\{1,2,3\},\{1,2,4\},\{1,3,4\}\}, \mathcal{M}(S)=\{2\}$ if $S \in\{\{2,3\},\{2,4\},\{2,3,4\}\}$ and $\mathcal{M}(S)=\emptyset$ otherwise one easily finds that $v^{c^{\Gamma}}=v_{\mathcal{M}, w}$. Now $\mathcal{M}$ is proper and veto-rich but not monotonic. From Theorem 3.5 we derive that $v^{c^{\Gamma}}$ is balanced and hence from Proposition 4.1 that $c^{\Gamma}$ is balanced. Although there is freedom in choosing $\mathcal{M}$ that generates the cost savings game $v^{c^{\Gamma}}$, no coalitional map $\mathcal{M}$ can be both monotonic and veto-rich. Otherwise, according to Theorem 3.6 and Proposition 4.1, the game $c^{\Gamma}$ would have a PMAS as well.

Let $\Gamma=(N, E)$ be a complete multipartite graph and let $\left\{P_{1}, P_{2}, \ldots, P_{r}\right\}$ be the corresponding partition of the vertex set $N$. We list the nodes in $N$ in some order $N=\left\{v_{1}, v_{2}, v_{3}, \ldots, v_{n}\right\}$, with $n=|N|$ (not yet having a cost vector $w \in \mathbb{R}_{+}^{N}$ in mind).

Let $[n]=\{1, \ldots, n\}$. For every $v_{i} \in N$, with $i \in[n]$, let $k\left(v_{i}\right) \in\{1, \ldots, r\}$ be such that $v_{i} \in P_{k\left(v_{i}\right)}$. Define the $\operatorname{map} \mathcal{M}$ by

$$
\mathcal{M}(S)=\left\{v_{i} \in S \mid \text { there is a } v_{j} \in S \cap P_{k\left(v_{i}\right)} \text { with } i, j \in[n] \text { and } j>i\right\}
$$

for every $S \in 2^{N}$. In other words, $\mathcal{M}(S)$ is formed by members of $S$ that precede in the order another member of $S$ in the same partition element. One easily verifies the following statement.

Proposition 4.29 Let $\Gamma=(N, E)$ be a complete multipartite graph and let $\mathcal{M}$ be the coalitional map as defined in (25). Then $\mathcal{M}$ is monotonic, proper and veto-rich but not necessarily supermodular (this is only true if all partition elements have at most two elements, see Remark 4.30).

Remark 4.30 Let $\Gamma=(N, E)$ be a complete multipartite graph and let $\left\{P_{1}, P_{2}, \ldots, P_{r}\right\}$ be the corresponding partition of the vertex set $N$ such that $\left|P_{l}\right| \leq 2$ for $l=1, \ldots, r$. We want to show that the coalitional map $\mathcal{M}$ defined by (25) on $\Gamma$ is supermodular. First, consider two coalitions $S, T \in 2^{N}$ such that $v_{i} \in \mathcal{M}(S) \cap \mathcal{M}(T), i \in[n]$. To be selected by both $\mathcal{M}(S)$ and $\mathcal{M}(T), v_{i}$ must be in both $S$ and $T$, and in both $S$ and $T$ there must be a $v_{j} \in P_{k\left(v_{i}\right)}$ with $j \in[n]$ and $j>i$. So, $v_{i}, v_{j} \in S \cap T$ and it follows that $v_{i} \in \mathcal{M}(S \cap T)$. Now, take $v_{i} \in \mathcal{M}(S \cap T)$ with $i \in[n]$. This means that $v_{i} \in S \cap T$ and there is a $v_{j} \in S \cap T \cap P_{k\left(v_{i}\right)}$ with $j \in[n]$ and $j>i$. So, $v_{i} \in \mathcal{M}(S) \cap \mathcal{M}(T)$ and we can conclude that $\mathcal{M}(S) \cap \mathcal{M}(T)=\mathcal{M}(S \cap T)$, i.e. $\mathcal{M}$ is supermodular.

On the other hand it is easy to check that if $\Gamma=(N, E)$ is a complete multipartite graph with some partition element $P_{k}$ such that $\left|P_{k}\right|>2$, then $\mathcal{M}$ is not necessarily supermodular. For instance, take $P_{k}=\left\{v_{h}, v_{i}, v_{j}\right\}$ with $h, i, j \in[n]$ and $h<i<j$, and let $S=\left\{v_{h}, v_{j}\right\}$ and $T=\left\{v_{h}, v_{i}\right\}$. Then $\mathcal{M}(S)=\left\{v_{h}\right\}$ and $\mathcal{M}(T)=\left\{v_{h}\right\}$, but $\mathcal{M}(S \cap T)=\mathcal{M}\left(\left\{v_{h}\right\}\right)=\emptyset$.

The following proposition is a direct consequence of Theorems 3.3, 3.4, 3.5, 3.6 and Proposition 4.29.

Proposition 4.31 Let $\Gamma=(N, E)$ be a complete multipartite graph and let $\mathcal{M}$ be the coalitional map as defined in (25). Then $\left(N, v_{\mathcal{M}, w}\right)$ is monotonic, superadditive, (totally) balanced and PMAS-admissible for every $w \in \mathbb{R}_{+}^{N}$.

Now, we will show that the cost savings games $v^{c^{\Gamma, w}}$, corresponding to the weighted minimum coloring games $c^{\Gamma, w}$ on complete multipartite graphs, form a subset of GAGs $\left(N, v_{\mathcal{M}, w}\right)$ with $\mathcal{M}$ specified by $(25)$. Let $\left(N, c^{\Gamma, w}\right)$ be the weighted minimum coloring game associated with a complete multipartite graph and cost vector $w \in \mathbb{R}_{+}^{N}$. Choose the ordering of the vertices in $N$ according to increasing costs, i.e. $N=\left\{v_{1}, v_{2}, v_{3}, \ldots, v_{n}\right\}$ such that $w_{v_{1}} \leq w_{v_{2}} \leq \cdots \leq w_{v_{n}}$, and let $\mathcal{M}$ be the corresponding coalitional map according to (25). Define the set $K(S)=$ $S \backslash \mathcal{M}(S)$ for every $S \in 2^{N}$. The set $K(S)$ is a maximum weighted clique in $\Gamma_{\mid S}$ with weight $\sum_{i \in[n]: v_{i} \in K(S)} w_{v_{i}}$, with $[n]=\{1, \ldots, n\}$, and this is also the weighted chromatic number of $\Gamma_{\mid S}$ with respect to $w_{\mid S}$ since $\Gamma$ is perfect. Therefore $c^{\Gamma, w}(S)=\sum_{i \in[n]: v_{i} \in K(S)} w_{v_{i}}$ for every $S \in 2^{N}$. So indeed we have

$$
v^{c^{\Gamma, w}}(S)=\sum_{i \in[n]: v_{i} \in S} c^{\Gamma, w}\left(\left\{v_{i}\right\}\right)-c^{\Gamma, w}(S)=\sum_{i \in[n]: v_{i} \in S} w_{v_{i}}-\sum_{i \in[n]: v_{i} \in K(S)} w_{v_{i}}=\sum_{i \in[n]: v_{i} \in \mathcal{M}(S)} w_{v_{i}}=v_{\mathcal{M}, w}(S)
$$

for every $S \in 2^{N}$.

Consequences of the characterizations in Chapter 3 for (weighted) minimum coloring games As a consequence of Proposition 4.31 we obtain the following. 
Proposition 4.32 Cost saving games corresponding to weighted minimum coloring games on complete multipartite graphs are monotonic, superadditive, (totally) balanced and PMAS-admissible.

Proposition 4.33 Weighted minimum coloring games on complete multipartite graphs are subadditive, (totally) balanced and PMAS-admissible.

Proof The proof follows directly from the properties of cost saving games corresponding to weighted minimum coloring games on complete multipartite graphs and by Proposition 4.1.

Some results on a particular class of (weighted) minimum coloring games

Weighted minimum coloring games corresponding to complete multipartite graphs are known to be submodular as well. In case all permutation elements have at most two elements, this follows from Remark 4.30, Theorem 3.7 and Proposition 4.1. For general complete multipartite graphs we can prove that (unweighted) minimum coloring games $\left(w_{i}=1\right.$ for every $\left.i \in N\right)$ are submodular as follows. For every $k \in\{1, \ldots, r\}$ let $p_{k}$ be the element of $P_{k}$ with the smallest index. Define the coalitional map $\mathcal{M}$ by

$$
\mathcal{M}(S)=\left\{p_{k} \mid k \in\{1, \ldots, r\}, P_{k} \subseteq S\right\}
$$

for every $S \in 2^{N}$. One can easily verifies the following proposition.

Proposition 4.34 Let $\Gamma=(N, E)$ be a complete multipartite graph and let $\mathcal{M}$ be the coalitional map as defined in (26). Then $\mathcal{M}$ is supermodular.

The following proposition is a direct consequence of Theorem 3.7 and Proposition 4.34.

Proposition 4.35 Let $\Gamma=(N, E)$ be a complete multipartite graph and let $\mathcal{M}$ be the coalitional map as defined in (26). Then $\left(N, v_{\mathcal{M}, w}\right)$ is supermodular for every $w \in \mathbb{R}_{+}^{N}$.

For every $S \in 2^{N}$ and $w \in \mathbb{R}_{+}^{N}$ we have $c^{\Gamma}(S)=\left|\left\{k \in\{1, \ldots, r\} \mid P_{k} \cap S \neq \emptyset\right\}\right|$. For the dual game $c^{\Gamma *}$ we have $c^{\Gamma *}(S)=c^{\Gamma}(N)-c^{\Gamma}(N \backslash S)=r-\left|\left\{k \in\{1, \ldots, r\} \mid P_{k} \cap(N \backslash S) \neq \emptyset\right\}\right|=\left|\left\{k \in\{1, \ldots, r\} \mid P_{k} \subseteq S\right\}\right|=\sum_{i \in \mathcal{M}(S)} w_{i}=$ $v_{\mathcal{M}, w}(S)$ for every $S \in 2^{N}$. As a consequence, we obtain the following.

Proposition 4.36 Dual games corresponding to minimum coloring games on complete multipartite graphs are supermodular.

Proof It follows from the fact that $c^{\Gamma *}=v_{\mathcal{M}, w}$ for every $w \in \mathbb{R}_{+}^{N}$, and in particular for the vector $w$ such that $w_{i}=1$ for every $i \in N$.

Proposition 4.37 Minimum coloring games corresponding to complete multipartite graphs are submodular.

Proof The proof follows directly from the properties of supermodularity of dual games corresponding to minimum coloring games on complete multipartite graphs and by Proposition 4.2.

Finally, let us consider a graph $\Gamma=(N, E)$ that is $\left(2 K_{2}, P_{4}\right)$-free. Moreover, let $w \in \mathbb{N}^{N}$ be such that $w_{i}=1$ for every $i \in N$. From [29] and [30] we know that $\Gamma$ has a rooted forest representation: there is a rooted forest $(N, F)$ such that for every $i, j \in N, i \neq j$ we have $\{i, j\} \notin E$ if and only if $i \in D(j)$ or $j \in D(i)$. Here for every $i \in N$ the set $D(i)$ denotes the unique path in $(N, F)$ from $i$ to the root of its tree (including $i$ and the root of the tree). Now define the map $\mathcal{M}$ by

$$
\mathcal{M}(S)=\{i \in S \mid \text { there is a } j \in S, j \neq i \text { with } i \in D(j)\}
$$

for every $S \in 2^{N}$. One easily verifies the following proposition.

Proposition 4.38 Let $\Gamma=(N, E)$ be a $\left(2 K_{2}, P_{4}\right)$-free graph and let $\mathcal{M}$ be the coalitional map as defined in (27). Then $\mathcal{M}$ is monotonic, proper and veto-rich.

The following proposition is a direct consequence of Theorems 3.3, 3.4, 3.5, 3.6 and Proposition 4.38. 
Proposition 4.39 Let $\Gamma=(N, E)$ be a $\left(2 K_{2}, P_{4}\right)$-free graph and let $\mathcal{M}$ be the coalitional map as defined in (27). Then $\left(N, v_{\mathcal{M}, w}\right)$ is monotonic, superadditive, (totally) balanced and PMAS-admissible for every $w \in \mathbb{R}_{+}^{N}$.

It is straightforward to check that $c^{\Gamma}(S)=|S \backslash \mathcal{M}(S)|$ for every $S \in 2^{N}$. Now, for the cost savings game $v^{c^{\Gamma}}$ corresponding to $c^{\Gamma}$ we have $v^{c^{\Gamma}}(S)=\sum_{i \in S} c^{\Gamma}(\{i\})-c^{\Gamma}(S)=\sum_{i \in S} w_{i}-\sum_{i \in S \backslash \mathcal{M}(S)} w_{i}=\sum_{i \in \mathcal{M}(S)} w_{i}=v_{\mathcal{M}, w}(S)$ for every $S \in 2^{N}$ (recall that we are now assuming $w_{i}=1$ for all $i \in N$ ). As a consequence of Proposition 4.39 we obtain the following.

Proposition 4.40 Cost saving games corresponding to minimum coloring games on $\left(2 K_{2}, P_{4}\right)$-free graphs are monotonic, superadditive, (totally) balanced and PMAS-admissible.

Proposition 4.41 Minimum coloring games on $\left(2 K_{2}, P_{4}\right)$-free graphs are subadditive, (totally) balanced and PMASadmissible.

Proof The proof follows directly from the properties of cost saving games corresponding to weighted minimum coloring games on complete multipartite graphs and by Proposition 4.1.

\section{Conclusions}

In this paper we have introduced some characterizations for large families of GAGs [9], and we have shown how these results can be used to analyze common features among distinct classes of TU games (in particular, within the family of OR games [6]). The results for GAGs provided in Section 3 apply to all vectors of nonnegative contributions. As a consequence, in some cases, the generality of coalitional maps prevent an exhaustive search of properties of the associated games. In this case, as shown for generalized airport games and also for link connection games, conditions for GAGs over subsets of contribution vectors (in particular, convex cones) are more effective, and deserve a deeper understanding.

\section{References}

[1] Aadithya, K. V., Ravindran, B., Michalak, T. P., Jennings, N. R. (2010) Efficient computation of the Shapley value for centrality in networks. In International Workshop on Internet and Network Economics, 1-13. Springer Berlin Heidelberg.

[2] Amer, R., Giménez, J. M. (2004) A connectivity game for graphs. Mathematical Methods of Operations Research, $60(3), 453-470$.

[3] Bilbao, J. M. (2000) Cooperative games on combinatorial structures. Kluwer Academic Publishers.

[4] Bjørndal, E., Koster, M. and Tijs, S. (2004) Weighted allocation rules for standard fixed tree games. Mathematical Methods of Operations Research, 59(2), 249-270.

[5] Bonzon, E., Maudet N., Moretti S. (2014) Coalitional games for abstract argumentation. In Proceedings of the 5th International Conference on Computational Models of Argument (COMMA'14), 201.

[6] Borm, P., Hamers, H., Hendrickx, R. (2001) Operations research games: A survey. Top, 9(2), 139-199.

[7] Brânzei, R., Fragnelli, V., Tijs, S. (2002) Tree-connected peer group situations and peer group games. Mathematical Methods of Operations Research, 55(1), 93-106.

[8] Branzei, R., Moretti, S., Norde, Tijs, S. (2004) The P-value for cost sharing in minimum cost spanning tree situations. Theory and Decision, 56(1-2), 47-61.

[9] Cesari, G., Lucchetti, R., Moretti, S. (2017) Generalized Additive Games. International Journal of Game Theory, 46(4), 919-939. 
[10] Deng, X., Ibaraki, T., Nagamochi, H., Zang, W. (2000) Totally balanced combinatorial optimization games. Mathematical Programming, 87(3), 441-452.

[11] Hamers, H., Miquel, S., Norde, H. (2014) Monotonic stable solutions for minimum coloring games. Mathematical Programming, 145(1-2), 509-529.

[12] Hamers, H., Horozoglu, N., Norde, H., Platz, T. (2019) On totally balanced, submodular and PMAS-admissible weighted minimum colouring games. Working paper.

[13] Lindelauf, R.H.A., Hamers, H.J.M., Husslage, B.G.M. (2013) Cooperative game theoretic centrality analysis of terrorist networks: The cases of jemaah islamiyah and al qaeda. European Journal of Operational Research, $229(1), 230-238$.

[14] Littlechild, S.C., Owen, G. (1973) A simple expression for the Shapley value in a special case. Management Science, 20, 370-372.

[15] Littlechild, S.C., Thompson, G.F. (1977) Aircraft landing fees: a game theory approach. Bell Journal of Economics, 8, 186-204.

[16] Moretti, S. (2018) On Cooperative Connection Situations Where the Players Are Located at the Edges. In: Belardinelli F., Argente E. (eds) Multi-Agent Systems and Agreement Technologies. EUMAS 2017 , AT 2017. Lecture Notes in Computer Science, vol 10767. Springer, Cham.

[17] Moretti, S., Norde, H. (2020) A short note on weighted multi-glove games. Technical report of the Paris Dauphine University, "CAHIER DU LAMSADE" n.394.

[18] Moretti, S., Norde, H., Pham Do, K.H., Tijs, S. (2002) Connection problems in mountains and monotonic allocation schemes. Top, 10(1), 83-99.

[19] Nagamochi, H., Zeng, D. Z., Kabutoya, N., Ibaraki, T. (1997) Complexity of the minimum base game on matroids. Mathematics of Operations Research, 22(1), 146-164.

[20] Norde, H., Fragnelli, V., García-Jurado, I., Patrone, F., Tijs, S. (2002) Balancedness of infrastructure cost games. European Journal of Operational Research, 136(3), 635-654.

[21] Norde, H., Moretti, S., Tijs, S. (2004) Minimum cost spanning tree games and population monotonic allocation schemes. European Journal of Operational Research, 154(1), 84-97.

[22] Okamoto, Y. (2003) Submodularity of some classes of the combinatorial optimization games. Mathematical Methods of Operations Research, 58(1), 131-139.

[23] Schrijver, A. (2003) Combinatorial Optimization. Springer-Verlag.

[24] Shapley, L.S., Shubik, M. (1969) Pure competition, coalitional power, and fair division. International Economic Review, 10, 337-362.

[25] Sprumont, Y. (1990) Population monotonic allocation schemes for cooperative games with transferable utility. Games and Economic Behavior, 2, 378-394.

[26] Suri, N.R., Narahari, Y. (2008). Determining the top-k nodes in social networks using the Shapley value. In Proceedings of the 7th international joint conference on Autonomous agents and multiagent systems, 3, $1509-1512$.

[27] Tijs, S., Branzei, R., Moretti, S., Norde, H. (2006) Obligation rules for minimum cost spanning tree situations and their monotonicity properties. European Journal of Operational Research, 175(1), 121-134.

[28] Wiese, H. (2004) The outside-option value-axiomatization and application to the gloves game. Mimeo.

[29] Wölk, E.S. (1965) A note on "the comparablity graph of a tree". Proceedings of the American Mathematical Society, 16, 17-20.

[30] Yan, J., Chen, J., Chang, G.J. (1996) Quasi-threshold graphs. Discrete Applied Mathematics, 69, $247-255$. 